\title{
Annealing synchronizes the $70 S$ ribosome into a minimum-energy conformation
}

\begin{abstract}
Authors: Xiaofeng $\mathrm{Chu}^{1,2 \dagger}$, Xin $\mathrm{Su}^{1 \dagger}$, Mingdong $\mathrm{Liu}^{1,3}, \mathrm{Li} \mathrm{Li}^{4}$, Tianhao $\mathrm{Li}^{1,3}$, Yicheng Qin ${ }^{1}$,
\end{abstract} Guoliang Lu ${ }^{4}$, Lei Qi ${ }^{2}$, Yunhui Liu ${ }^{1}$, Jinzhong Lin ${ }^{4}$, Qing-Tao Shen ${ }^{1,2 *}$

\author{
Affiliations: \\ ${ }^{1}$ iHuman Institute and School of Life Science and Technology, ShanghaiTech University, \\ Shanghai 201210, China. \\ ${ }^{2}$ Laboratory for Marine Biology and Biotechnology, Qingdao National Laboratory for Marine \\ Science and Technology, Qingdao 266237, China. \\ ${ }^{3}$ University of Chinese Academy of Sciences, Beijing 100049, China. \\ ${ }^{4}$ State Key Laboratory of Genetic Engineering, School of Life Sciences, Zhongshan Hospital, \\ Fudan University, Shanghai, 200438. \\ †These authors contributed equally to this work. \\ ${ }^{*}$ Corresponding author. Email: shenqt@shanghaitech.edu.cn
}

\begin{abstract}
Researchers commonly anneal metals, alloys, and semiconductors to repair defects and improve microstructures via recrystallization. Theoretical studies indicate simulated annealing on biological macromolecules helps predict the final structures with minimum free energy. Experimental validation of this homogenizing effect and further exploration of its applications are fascinating scientific questions that remain elusive. Here, we chose the apo-state $70 S$ ribosome from Escherichia coli as a model, wherein the $30 S$ subunit undergoes a thermally driven inter-subunit rotation and exhibits substantial structural flexibility as well as distinct free energy. We experimentally demonstrate that annealing at a fast cooling rate enhances the $70 S$ ribosome homogeneity and improves local resolution on the $30 S$ subunit. After annealing, the $70 S$ ribosome is in a nonrotated state with respect to corresponding intermediate structures in unannealed or heated ribosomes, and exhibits a minimum energy in the free energy landscape. One can readily crystallize these minimum-energy ribosomes, which have great potential for synchronizing proteins on a single-molecule level. Our experimental results are consistent with
\end{abstract}


theoretical analysis on the temperature-dependent Boltzmann distribution, and offer a facile yet robust approach to enhance protein stability, which is ideal for high-resolution cryogenic electron microscopy. Beyond structure determination, annealing can be extended to study protein folding and explore conformational and energy landscape.

\section{Significance statement}

In metallurgy, annealing heats a metal or alloy to a predetermined temperature, holding for a certain time, and then cooling to room temperature to change the physical and sometimes also the chemical properties of the material. Researchers introduce the similar concept as simulated annealing to predict minimum-energy conformations of biological macromolecules. In this work, we experimentally verify that annealing at a fast cooling rate can synchronize the $70 \mathrm{~S}$ ribosome into a nonrotated state with a minimum energy in the free energy landscape. Our results not only offer a facile yet robust approach to stabilize proteins for high-resolution structural analysis, but also contribute to the understanding of protein folding and temperature adaptation. 


\section{INTRODUCTION}

Annealing - common in metallurgy - heats a metal or alloy to a set temperature, holds this temperature, and then cools to room temperature to improve the physical and sometimes also the chemical properties of the material $(1,2)$. During annealing, recrystallization repairs defects to afford a refined microstructure, which can relieve stress, soften the metal, increase the ductility, and improve the grain structure. One can also anneal organic/inorganic semiconductors via rearranging molecules, polymer chain segments, or entire polymer chains into active layer films. Optimizing the annealing temperature and time can either dramatically increase the crystal size from the sub-micrometer scale to several micrometers, or yield different crystals (3-5).

Annealed materials tend to adopt homogenous states and readily assemble into either three-dimensional (3D) or two-dimensional (2D) crystals. One can readily visualize such regular packing via atomic force microscopy (AFM), X-ray diffraction (XRD), or electron microscopy (EM). Whether annealing exhibits similar effects on biological macromolecules, especially proteins, is a fascinating scientific question that remains unanswered. To date, annealing on proteins has mainly been limited to molecular dynamics simulations. Researchers have simulated annealing - such as with Monte Carlo algorithms-on proteins from a set of starting conformations, which has helped predict the final structures with minimum free energy (6-8). Distinct from metals and organic polymers, proteins and protein complexes are usually discrete entities that consist of chemically diverse subunits bound together in diverse geometries. This substantial structural heterogeneity hinders direct structural determination via AFM or XRD. In contrast, recent advancements in the resolution of cryo-EM offer great opportunities to obtain high-resolution protein structures at the single-molecule level (9-12). By using cryo-EM to compare detailed structures before/after annealing, one can obtain direct experimental evidence for annealing to affect protein conformations.

Many proteins, especially protein complexes, can in principle exhibit diverse conformations. Based on the Boltzmann distribution formula, each conformational state of a protein or protein complex is occupied with a statistical weight, $P_{i}=e^{-\left(E_{i} / k_{B} T\right)}$, where $E_{i}$ is the free energy of the conformational state $i, k_{B}$ is the Boltzmann constant, and $T$ is the absolute temperature. The Boltzmann distribution and the derived free energy are temperature-dependent; an increase in temperature can directly increase the free energy and help a protein escape the 
energy barrier between local minima. At room and physiological temperatures, minimum-energy conformations will usually differ from those at lower temperatures. This is the theoretical basis for protein conformational changes in accordance with temperature.

\section{RESULTS}

\section{Annealing improves local resolution}

Here, we chose the apo-state $70 S$ ribosome from Escherichia coli as a model, wherein the $30 S$ subunit undergoes a thermally driven inter-subunit rotation (13-18) and exhibits substantial structural flexibility as well as distinct free energy (19). We incubated purified apo-state $70 S$ ribosome at $0^{\circ} \mathrm{C}$ for $5 \mathrm{~min}$, then immediately flash-froze the ribosome for cryo-EM analysis, which retained the same conformation as before vitrification (depicted as the unannealed state). We screened the collected $70 S$ ribosome particles via discarding obvious junk and disassembled ribosomes through 2D and 3D classifications. Reconstruction from 200,000 randomly selected particles yielded a structure at a final resolution of $2.6 \AA$, based on gold-standard Fourier shell correlation (FSC; Fig. 1A and figs. S1-3). Due to the lack of stabilizing factors, such as messenger and transfer RNA, local resolution estimates on the unannealed $70 S$ ribosome indicated variable resolutions across the entire density map over the 2.6-7.2 $\AA$ range (Fig. 1A). Relative to the $50 S$ subunit, the $30 S$ subunit-especially its head domain-was less wellresolved, which is common in other apo-state ribosomes $(20,21)$.

To quantitate the resolution variation of different regions, we calculated the local resolution via averaging local resolution values within the selected regions (figs. S4-6). Our analysis indicated that the $50 S$ subunit exhibited an average local resolution of $3.1 \AA$, whereas the $30 S$ subunit was much less resolved - only $5.2 \AA$. Furthermore, the $30 S$ head domain was even less resolved - average resolution of $6.1 \AA$ (Fig. 1B). The inter-subunit ratcheting between the $50 S$ and $30 S$ subunits is the primary cause for poor resolvability; the intra-subunit swirling of the $30 S$ subunit is secondary, which lessens the resolution of the head domain. For simplicity, we used the local resolution of the $30 S$ subunit as a marker to monitor the effect of annealing on the $70 S$ ribosome. 
Full annealing consists of two tandem heating and cooling steps. Cooling rate and annealing temperature are usually two critical elements for homogenization of metals, alloys, and semiconductors $(22,23)$. In metallurgy, the annealing temperature is usually $30^{\circ} \mathrm{C}$ to $50^{\circ} \mathrm{C}$ greater than the upper critical temperature of metals (24). In molecular dynamics simulation, researchers sometimes use extreme annealing temperatures - such as $127^{\circ} \mathrm{C}$ - to help biological macromolecules escape energy barriers. To avoid heat-induced denaturation, we set the annealing temperatures of the $70 \mathrm{~S}$ ribosome below the $\sim 72^{\circ} \mathrm{C}$ melting temperature of bacterial ribosomes $(25,26)$. We carried out subsequent cooling on heated ribosomes in highly thermoconductive PCR tubes, via either gradual cooling in the PCR machine or immediate immersion into an ice bath. We vitrified the annealed samples in a Vitrobot chamber preset at $4{ }^{\circ} \mathrm{C}$, and then subjected the samples to single-particle cryo-EM analysis in accordance with the same procedure used for unannealed ribosomes from the exact same number $(200,000)$ of randomly selected particles (Fig. 2A).

We performed systematic screening of various combinations of cooling rates and annealing temperatures via comparing the local resolutions of the $30 S$ subunit. When we gradually cooled the $70 \mathrm{~S}$ ribosome over the course of $2 \mathrm{~h}$ from $37^{\circ} \mathrm{C}$ to $0^{\circ} \mathrm{C}$, the average local resolution of the $30 S$ subunit was $5.1 \AA$, and there was no obvious local resolution improvement with respect to the unannealed ribosome-5.2 $\AA$ (Fig. 2B, C and fig. S6). Fast cooling via immersion into an ice bath $(<1 \mathrm{~s})$ improved the local resolution from a value of $5.2 \AA$ to a value of $\sim 4.0 \AA$ on the $30 S$ subunit (Fig. 2B, C and fig. S6). A mixture of salt, ice, and watercomparatively high thermo-conductivity-yielded improved local resolution of the $30 \mathrm{~S}$ subunit-3.7 $\AA$; the $30 S$ head was also dramatically improved to $4.4 \AA$, relative to $6.1 \AA$ from the unannealed ribosome (Figs. 1B and 2B, C and fig. S6). Apparently, one can use fast instead of gradual cooling to improve the local resolution of the $30 S$ subunit. As another critical parameter for annealing in metallurgy, annealing temperatures of $45^{\circ} \mathrm{C}, 55^{\circ} \mathrm{C}$, and $65^{\circ} \mathrm{C}$ did not further improve local resolutions of the $30 \mathrm{~S}$ subunit, compared with $30^{\circ} \mathrm{C}$ and $37^{\circ} \mathrm{C}$ (Fig. $2 \mathrm{~B}$, $\mathrm{C}$ and fig. S6). Thus, we considered fast cooling from $37^{\circ} \mathrm{C}$ in a mixture of salt, ice, and water as ideal annealing conditions for improving the local resolution of the $70 S$ ribosome.

To further understand the annealing process, we also investigated heating the $70 \mathrm{~S}$ ribosome to an annealing temperature of $37^{\circ} \mathrm{C}$ as an intermediate state by cryo-EM (Fig. 2A). Researchers recently developed temperature-resolved cryo-EM to study temperature-sensitive 
proteins, such as transient receptor potential vanilloid-3 (TRPV3) and ketol-acid reductoisomerase (KARI), at optimal temperatures $(27,28)$. We optimized sample preparation, such as by consistent high-temperature control and efficient prevention of ice contamination, and found these procedures useful for obtaining ideal cryo-EM grids (fig. S7 and movie S1). We resolved 200,000 particles of the heated $70 S$ ribosome at a resolution of $2.7 \AA$, close to that of the unannealed ribosome. However, the average local resolution of the $30 S$ subunit was $6.0 \AA$, substantially less than that of the unannealed and annealed structures (Figs. 1B and 2B, C and fig. S6). The $30 S$ head domain was only resolved at $7.2 \AA$, and the densities were almost indiscernible at the threshold level proper to the $50 S$ subunit (Figs. 1 and 2B, C and fig. S6). Thus, heating increased the free energy of the $70 \mathrm{~S}$ ribosome and imparted more structural flexibility to the $30 S$ subunit, which to some extent hindered final reconstruction and afforded

\section{Annealing stabilizes flexible regions}

Relative to the unannealed $70 S$ ribosome, the overall structure of the annealed $70 S$ ribosome exhibited substantially higher resolution. The resolution improvement was not uniform across the entire $70 S$ ribosome. For instance, the $30 S$ subunit, which was poorly resolved in the unannealed ribosome, was dramatically improved $1.5 \AA$-i.e., from a value of $5.2 \AA$ to a value of $3.7 \AA$. As the control, the well-resolved $50 S$ subunit was only enhanced $0.3 \AA$-i.e., from a value of $3.1 \AA$ to a value of $2.8 \AA$-after annealing (Fig. 1B). Thus, annealing was especially beneficial to low-resolution regions, which have greater structural flexibility. To further verify this deduction, we performed a comprehensive statistical analysis on the average local resolution of the same sub-regions between the unannealed and annealed $70 \mathrm{~S}$ ribosomes. For example, annealing improved the average local resolutions of different regions at the level of $\sim 0.1,0.6,0.8$, 1.2, and $2.0 \AA$; the corresponding regions in the unannealed ribosome had local resolution in the range of 2.5-3.0, 3.0-3.5, 4.0-4.5, 5.0-5.5, and 5.5-6.0 А (Fig. 3A). An exponential curve fit well to the data, indicating that more flexibility in the unannealed $70 S$ ribosome corresponded to more improvement in local resolution after annealing.

Rigid regions were well-resolved in both unannealed and annealed $70 S$ ribosomes, and most RNA fragments of $23 S$ rRNA and ribosomal proteins in the $50 S$ subunit were clearly 
resolved in the cryo-EM maps (Fig. 3B, C). There were some flexible peripheral regions in the $50 S$ subunit, which were poorly resolved in the unannealed ribosome. These regions were substantially more distinct after annealing (Fig. 1A). For instance, the L1 stalk, a mobile domain of the $50 \mathrm{~S}$ subunit that shields the exit site of the deacylated tRNA, is usually invisible in X-ray crystallography and cryo-EM, especially in the absence of peptidyl-tRNA and aminoacyl-tRNA (29-31). After annealing, the L1 stalk was resolved at a resolution of $4.9 \AA$, and adopted an orientation between the open and closed states, which researchers classify as an intermediate state during translation elongation (Fig. 3D-F). In addition to the L1 stalk, regions from 1,0341,121 and 1,437-1,553 in $23 S$ rRNA are intrinsically flexible, such that they interact with EF-GGTP (during elongation and termination procedures) and EF-Tu-GTP-aminoacyl-tRNA (help coupling with the SecY complex). The resolution of these flexible regions was substantially improved: from a value of $4.9 \AA$ to a value of $4.1 \AA$, and from a value of $4.0 \AA$ to a value of 2.9 $\AA$, respectively, after annealing (fig. S8).

Another resolution improvement after annealing was evident on the flexible $30 S$ subunit. In the annealed ribosome, the $30 S$ body domain was improved $1.3 \AA$ : i.e., from a value of $4.6 \AA$ to a value of $3.3 \AA$ (Figs. $1 \mathrm{~B}$ and $2 \mathrm{~B}$ ). Furthermore, swirling of the $30 S$ head domain, with respect to the $30 S$ body domain, imparted more structural flexibility to the $30 S$ head domain; we resolved the unannealed $30 S$ head domain to only $6.1 \AA$. After annealing, we dramatically stabilized the $30 S$ head domain and improved the resolution to $4.4 \AA$ (Figs. 1B and $2 \mathrm{~B}$ ). The resolution improvement in the $30 S$ subunit was also in accordance with our previous finding that greater structural flexibility corresponded to greater resolution improvement after annealing.

Compared with the unannealed ribosome, the overall structure of the heated ribosome was less well-resolved. The resolution loss also occurred mainly on the flexible regions of the $70 S$ ribosome. Similar local resolution comparisons between the unannealed and heated ribosomes indicated that more flexibility corresponded to a greater extent of resolution loss after heating. Briefly, the stable $50 S$ subunit in the heated $70 S$ ribosome had a similar resolution, at $\sim 3.3 \AA$, to the unannealed ribosome; whereas the flexible $30 S$ subunit was substantially less wellresolved, from 5.2-6.0 $\AA$ (Figs. 1B and 2B and fig. S8). Apparently, heating had a more substantial influence on the flexible rather than rigid regions, analogous to the effect of annealing. At low-resolution ranges, such as 5-6 $\AA$, in the unannealed ribosome, the resolution improvement via heating was $\sim 1 \AA$. This was not as large as the $\sim 2-\AA$ improvement upon 
annealing, which indicates that protein structure may be more sensitive to annealing than heating (Fig. 3A).

\section{Annealing renders a different state}

In metallurgy, recrystallization repairs defects and refines the microstructures after annealing. Thus, we tested whether the annealed $70 S$ ribosome underwent conformational changes. To sample such changes before/after annealing, one requires accurate modelingespecially on the $30 S$ subunit. We separately fit the $50 S$ and $30 S$ subunits from the crystallographic model (PDB ID 5MDZ) into the unannealed, heated, and annealed cryo-EM density maps via rigid body docking. Even though the fitting of the $30 \mathrm{~S}$ head domain into the unannealed and heated $70 \mathrm{~S}$ ribosomes was not as ideal as that of the annealed $70 \mathrm{~S}$ ribosome, the well-resolved $30 S$ body domains ensured docking accuracy among the unannealed, heated, and annealed $70 S$ ribosomes (fig. S9).

We aligned the unannealed, heated, and annealed $70 S$ ribosomes using the $50 S$ subunit as a reference. We compared the orientations of $30 S$ subunits via calculating the rotation angles relative to each other. The annealed $30 \mathrm{~S}$ subunit rotated clockwise $7.4^{\circ}$-relative to the unannealed $30 S$ subunit - exhibiting a distinct orientation. The motions of the $30 S$ subunit after annealing resulted in shifts at the periphery of the ribosome at $12.0 \AA$ (Fig. 4A). Annealing imparted a new orientation to the $70 \mathrm{~S}$ ribosome and rendered the $30 \mathrm{~S}$ subunit into a new state. The orientations of the $30 S$ subunits between the unannealed and heated $70 S$ ribosomes were close, with a variation less than $1.8 \AA$ (Fig. 4B). This further indicates that the annealing-induced orientation change occurs during cooling instead of heating.

Ratcheting of the $30 S$ subunit relative to the $50 S$ subunit is the major rotation that facilitates tRNA translocation. Researchers thoroughly investigated the nonrotated (PDB ID 4V9D) and fully rotated (PDB ID 4V7C) states of the $30 S$ subunit in the context of nascent peptide synthesis $(32,33)$. We aligned the unannealed, heated, and annealed ribosomes with both the nonrotated and fully rotated states, using the $50 S$ subunits as a reference. In the absence of mRNA, tRNA, and other factors, the unannealed 30S subunit adopted an intermediate state between the nonrotated and fully rotated states. Specifically, the unannealed $30 S$ subunit reached the fully rotated state via a $5.4^{\circ}$ anticlockwise rotation, and clockwise rotated $5.2^{\circ}$ to remain in a 
nonrotated state. The motion distance from the unannealed $30 S$ subunit to the nonrotated and fully rotated states was 10.5 and $8.2 \AA$, respectively (Fig. 4C). The heated $30 S$ subunit exhibited a similar orientation to the unannealed $30 S$ subunit and remained in the intermediate state between the nonrotated and fully rotated states (Fig. 4A, C). Distinct from the unannealed and heated $30 S$ subunits, the annealed $30 S$ subunit adopted an orientation very close to the nonrotated state, with a rotation angle of $2.2^{\circ}$ and a maximum motion distance of $5.4 \AA$ (Fig. 4D). The slight difference may be attributable to gentle swirling of the $30 S$ head domain. Apparently, annealing switched the $30 S$ subunit from an intermediate state to a nonrotated state (Fig. 4E), which is an appropriate state for a new ribosome cycle (34).

The $30 S$ subunits under most annealing conditions adopted very similar orientations as the standard annealing condition in the nonrotated states (fig. S10A-F). Even when we extended the hold time after annealing to $24 \mathrm{~h}$ at $0^{\circ} \mathrm{C}$, we resolved the $30 S$ subunit at an average resolution

of $3.9 \AA$ and the subunit remained in the nonrotated state, which indicated that the nonrotated state of the $30 S$ subunit may be an energetically favorable state. The exception is that when we slowly cooled the heated $70 \mathrm{~S}$ ribosome from a value of $37^{\circ} \mathrm{C}$ to a value of $4^{\circ} \mathrm{C}$ over $2 \mathrm{~h}$ in the PCR machine, the orientation of the $30 S$ subunit was similar to the unannealed and heated ribosomes in the intermediate state (fig. S10G). Considering that the local resolution of the $30 \mathrm{~S}$

\section{Annealing minimizes the free energy}

We used free-energy landscape analysis to investigate the correspondence between the subunit from a slowly cooled ribosome was substantially inferior compared with the annealed ribosome, the new orientation imparted by annealing was relevant to the resolution improvement. annealing-induced resolution improvement and conformational change. As the major conformational change during polypeptide synthesis, inter-subunit ratcheting between the $50 S$ and $30 S$ subunits was our proxy to monitor the difference between the unannealed and annealed $70 S$ ribosomes. To align the intermediate states, we merged 100,000 particles from the unannealed and 100,000 particles from the annealed ribosomes with different labels into one dataset, and we subjected the merged dataset to a manifold-based analysis in the same conformational coordinate as described elsewhere (35). In the projection direction approximately orthogonal to the interface between the $50 S$ and $30 S$ subunits, the manifold indicated two clusters, 
wherein particles from the annealed and unannealed $70 S$ ribosomes were well-separated from each other (Fig. 5A, movie S2). This result corresponds to the obvious orientation difference between the unannealed and annealed $70 S$ ribosomes.

We classified the merged particles into 50 frames based on the orientation of the projections, and we separately calculated the particle number of each frame via the labels corresponding to either the unannealed or annealed ribosomes. The unannealed and annealed $70 S$ ribosomes exhibit different peak positions along the frame trajectory. The annealed ribosome was mainly featured in the first 25 frames, whereas the unannealed ribosome started from frame 20 (Fig. 5B). Apparently, the unannealed and annealed ribosomes have distinct particle distributions along the frame trajectory. We reconstructed particles of each frame into a 3D structure with a resolution ranging from $\sim 5.0-10.0 \AA$, and we fit the respective atomic models for each frame via rigid body docking of the $50 S$ and $30 S$ subunits. As the entire body, we aligned the $70 S$ ribosome from different frames based on the $50 S$ subunit, and we calculated the rotation angles of the $30 S$ subunit in different frames relative to the nonrotated state. The rotation angles from frames 1-30 were limited within an ultra-small range of $1.5^{\circ}$, a range that included more than $55 \%$ of the particles (the ratio to the total 100,000 particles) of the annealed ribosome. From frames $31-50$, the rotation angles of $30 S$ subunit were $\sim 5.0^{\circ}$, a range that included $\sim 12 \%$ particles in frames $1-30$ and $36 \%$ in frames $31-50$.

We recalculated the particle distribution of the unannealed and annealed ribosomes against the rotation angles at a step size of $0.2^{\circ}$, and we conducted a moving average to smooth data variations. In the new particle distribution curve, the annealed ribosomes exhibited a sharp peak, which was closer to the nonrotational state; a rotation angle of $0^{\circ}$ (Fig. 5C). The sharp distribution indicated improved local resolution with stronger structural rigidity of the annealed ribosome. As the control, the unannealed ribosome exhibited no obvious peaks across the rotation angles from $0^{\circ}$ to $8.0^{\circ}$. The wider distribution inevitably deteriorated the local resolution of reconstruction, which fits well with the lower resolution in the unannealed $30 S$ subunit.

Based on the Boltzmann distribution, we calculated the free energy of various conformations, including both unannealed and annealed ribosomes, against the rotation angles of the $30 S$ subunit. Accordingly, the annealed ribosome exhibited a narrower and deeper well with a 
minimum energy, whereas the unannealed ribosome exhibited a wider and swallower well. Based on our experimental data, annealing led the $70 \mathrm{~S}$ ribosome from a wider and swallower well (local minima) to a narrower and deeper well (minimum energy state, global minima), and dramatically improved the resolution (Fig. 5D).

Deduced from the Boltzmann distribution formular, one can integrate all atomic arrangements within one conformational state as follows:

$$
P(A)=\int_{x \in A} P(x) \propto \int_{x \in A} \exp \left(-E(x) / k_{B} T\right) d x,(1)
$$

where $P(A)$ is the probability to remain in conformation $A$. Proteins at lower temperatures have a higher probability of remaining in a narrower and deeper well with a minimum-energy state compared with higher temperatures. Our data quantitatively agrees between predictive theory and experiments and provides direct evidence that annealing synchronizes the $70 \mathrm{~S}$ ribosome at a single-molecule level.

Annealing synchronized the $70 S$ ribosome into the homogeneous nonrotated state with minimum energy. We speculate that such ribosomes readily assemble into crystals, as in metallurgy. Actually, crystallization of ribosomes/nucleosomes features an annealing-like treatment, wherein researchers usually warm ribosomes/nucleosomes to between $37^{\circ} \mathrm{C}$ and $55^{\circ} \mathrm{C}$, then decrease to room temperature $\left(19^{\circ} \mathrm{C}\right)(36,37)$. To test assembly into crystals, we performed strict annealing on the $70 \mathrm{~S}$ ribosome and cooled the annealed ribosome to $4^{\circ} \mathrm{C}$ for crystallization (Fig. 5E). The unannealed ribosome and the slowly cooled ribosome were controls. Statistical analysis showed that the annealed ribosomes formed more crystals than the unannealed and slowly cooled ribosomes. Annealed ribosomes remained in a more homogeneous minimumenergy state, which facilitated assembly into crystals (Fig. 5F).

As the intermediate state of the annealing, we investigated the structural switch from a heated ribosome to an annealed ribosome via free-energy landscape analysis on the combined heated and annealed ribosomes. Similar to the combination of unannealed and annealed ribosomes, heated and annealed ribosomes also exhibited similar particle distribution patterns (fig. S11A-D). As the control, when we analyzed the unannealed and heated ribosomes, the particle distribution was similar, and the heated ribosome had an even wider distribution (fig. $\mathrm{S} 11 \mathrm{E})$. These results indicate that the conformational change occurred during cooling. 


\section{DISCUSSION}

Researchers widely use annealing on metals, alloys, and semiconductors to decrease the number of defects in crystals and increase the size of the crystals. Here, we experimentally demonstrated that annealing can also synchronize the $70 \mathrm{~S}$ ribosome into the minimum-energy state on a single-molecule level (Fig. 6). After annealing, the $70 S$ ribosome readily assembled into 3D crystals, which will inspire researchers to further investigate the physical and chemical effects of annealing on the $70 S$ ribosome and other biological macromolecules.

Structural flexibility, albeit critical for protein function, hinders researchers' ability to apply structural studies to elucidate function on a molecular level. Sustained efforts - such as mutations on key residues $(38,39)$, introduction of additional disulfide bonds (40, 41), addition of antibody/binding proteins (42-44), or cross-linking in a solution or within a glycerol/glucose gradient $(45,46)$ - have been useful for optimizing samples to increase structural stability. However, such efforts are time-consuming and can lack clear direction, and the final structures are confined to fixed states and sometimes are even distorted after additional manipulations. Annealing - a combination of appropriate heating and cooling - is non-destructive to proteins, and is a facile yet robust approach for high-resolution cryo-EM.

Researchers have also attempted to improve resolution via local refinement on flexible regions during cryo-EM image processing (47-50). We performed local refinements on the flexible $30 S$ subunit on both unannealed and annealed ribosomes. The average local resolution of the $30 S$ subunit of the unannealed $30 S$ subunit was improved $\sim 1 \AA$ to a value of $4.2 \AA$ after local refinements (figs. S12 and S13). Distinct from the resolution improvement via annealing, local refinement in itself still resulted in an insufficient average resolution of $5.5 \AA$ on the head domain of the $30 S$ subunit (figs. S12 and S13). Apparently, annealing and local refinement improve local resolution via different mechanisms. Annealing can drive biological macromolecules into a minimum-energy state and globally increase the resolution across the entire map, irrespective of the region size. As a control, local refinement worked on an algorithmic level and was only applicable to regions with reasonable sizes. When we applied local refinement to the annealed ribosome, both the body and head domains of the $30 \mathrm{~S}$ subunit were improved to 2.9 and $3.9 \AA$, respectively (figs. S12 and S13). This indicates that annealing is 
349 compatible with local refinement on flexible regions and can further optimize local resolutions 350 for detailed structural analysis.

351 One can use annealing to synchronize proteins into a minimum-energy state. Analogous 352 to cell synchronization, protein/conformation synchronization on a single-molecule level may be 353 helpful for many single-molecule approaches, such as those which use optical tweezers and 354 single-molecule fluorescence resonance energy transfer (51-53). One can also use annealing to 355 investigate temperature adaptation and protein folding, and to facilitate algorithm development in 356 molecular dynamics simulation. Thus, researchers should thoroughly investigate the mechanism 357 of annealing and further optimize the annealing conditions for improved resolution. 


\section{References and Notes:}

1. X. Huang, H. Sun, J. Shen, K. Cui, G. Wang, Different Effects of Annealing on Microstructure Evolution and SERS Performance for Cu-Cr Alloy Film and Bulk Alloy. Materials (Basel) 12 (2019).

2. C. Zhang et al., Effects of Annealing on Microstructure and Mechanical Properties of Metastable Powder Metallurgy CoCrFeNiMo0.2 High Entropy Alloy. Entropy (Basel) 21 (2019).

3. X. Yang, J. K. J. van Duren, R. A. J. Janssen, M. A. J. Michels, J. Loos, Morphology and Thermal Stability of the Active Layer in Poly(p-phenylenevinylene)/Methanofullerene Plastic Photovoltaic Devices. Macromolecules 37, 2151-2158 (2004).

4. Z. Zhu et al., Critical Annealing Temperature for Stacking Orientation of Bilayer Graphene. Small 14, e1802498 (2018).

5. R. M. Balagula et al., Effects of thermal annealing on localization and strain in core/multishell GaAs/GaNAs/GaAs nanowires. Sci Rep 10, 8216 (2020).

6. S. Cheon, F. Liang, Folding small proteins via annealing stochastic approximation Monte Carlo. Biosystems 105, 243-249 (2011).

7. M. Schmitz, G. Steger, Description of RNA folding by "simulated annealing". J Mol Biol 255, 254-266 (1996).

8. K. C. Chou, L. Carlacci, Simulated annealing approach to the study of protein structures. Protein Eng 4, 661-667 (1991).

9. M. Liao, E. Cao, D. Julius, Y. Cheng, Structure of the TRPV1 ion channel determined by electron cryo-microscopy. Nature 504, 107-112 (2013).

10. X. C. Bai, I. S. Fernandez, G. McMullan, S. H. Scheres, Ribosome structures to nearatomic resolution from thirty thousand cryo-EM particles. Elife 2, e00461 (2013).

11. T. Nakane et al., Single-particle cryo-EM at atomic resolution. Nature 587, 152-156 (2020).

12. K. M. Yip, N. Fischer, E. Paknia, A. Chari, H. Stark, Atomic-resolution protein structure determination by cryo-EM. Nature 587, 157-161 (2020).

13. P. V. Cornish, D. N. Ermolenko, H. F. Noller, T. Ha, Spontaneous intersubunit rotation in single ribosomes. Mol Cell 30, 578-588 (2008).

14. B. S. Schuwirth et al., Structures of the bacterial ribosome at 3.5 A resolution. Science 310, 827-834 (2005).

15. A. Vila-Sanjurjo et al., X-ray crystal structures of the WT and a hyper-accurate ribosome from Escherichia coli. Proc Natl Acad Sci U S A 100, 8682-8687 (2003).

16. W. Zhang, J. A. Dunkle, J. H. Cate, Structures of the ribosome in intermediate states of ratcheting. Science 325, 1014-1017 (2009).

17. T. Ahmed, J. Shi, S. Bhushan, Unique localization of the plastid-specific ribosomal proteins in the chloroplast ribosome small subunit provides mechanistic insights into the chloroplastic translation. Nucleic Acids Res 45, 8581-8595 (2017).

18. V. Jha et al., Structural basis of sequestration of the anti-Shine-Dalgarno sequence in the Bacteroidetes ribosome. Nucleic Acids Res 10.1093/nar/gkaa1195 (2020).

19. N. Fischer, A. L. Konevega, W. Wintermeyer, M. V. Rodnina, H. Stark, Ribosome dynamics and tRNA movement by time-resolved electron cryomicroscopy. Nature 466, 329-333 (2010). 
20. R. K. Flygaard, N. Boegholm, M. Yusupov, L. B. Jenner, Cryo-EM structure of the hibernating Thermus thermophilus $100 \mathrm{~S}$ ribosome reveals a protein-mediated dimerization mechanism. Nat Commun 9, 4179 (2018).

21. E. L. Murphy et al., Cryo-electron microscopy structure of the $70 \mathrm{~S}$ ribosome from Enterococcus faecalis. Sci Rep 10, 16301 (2020).

22. P. Divya et al., Rapid and Scalable Wire-bar Strategy for Coating of TiO2 Thin-films: Effect of Post-Annealing Temperatures on Structures and Catalytic Dye-Degradation. Molecules 25 (2020).

23. A. Bhaskar, H. Y. Chang, T. H. Chang, S. Y. Cheng, Effect of microwave annealing temperatures on lead zirconate titanate thin films. Nanotechnology 18, 395704 (2007).

24. R. Singh, "Chapter 12 - Heat Treatment of Steels" in Applied Welding Engineering, R. Singh, Ed. (Butterworth-Heinemann, Boston, 2012), https://doi.org/10.1016/B978-0-12391916-8.00012-1, pp. 95-108.

25. B. Pace, L. L. Campbell, Correlation of maximal growth temperature and ribosome heat stability. Proc Natl Acad Sci US A 57, 1110-1116 (1967).

26. P. Cammarano et al., Secondary structure features of ribosomal RNA species within intact ribosomal subunits and efficiency of RNA-protein interactions in thermoacidophilic (Caldariella acidophila, Bacillus acidocaldarius) and mesophilic (Escherichia coli) bacteria. Biochim Biophys Acta 740, 300-312 (1983).

27. C. Y. Chen, Y. C. Chang, B. L. Lin, C. H. Huang, M. D. Tsai, Temperature-Resolved Cryo-EM Uncovers Structural Bases of Temperature-Dependent Enzyme Functions. $J$ Am Chem Soc 141, 19983-19987 (2019).

28. A. K. Singh et al., Structural basis of temperature sensation by the TRP channel TRPV3. Nat Struct Mol Biol 26, 994-998 (2019).

29. S. Tishchenko et al., High-resolution crystal structure of the isolated ribosomal L1 stalk. Acta Crystallogr D Biol Crystallogr 68, 1051-1057 (2012).

30. P. V. Cornish et al., Following movement of the L1 stalk between three functional states in single ribosomes. Proc Natl Acad Sci US A 106, 2571-2576 (2009).

31. J. Fei, P. Kosuri, D. D. MacDougall, R. L. Gonzalez, Jr., Coupling of ribosomal L1 stalk and tRNA dynamics during translation elongation. Mol Cell 30, 348-359 (2008).

32. J. A. Dunkle et al., Structures of the bacterial ribosome in classical and hybrid states of tRNA binding. Science 332, 981-984 (2011).

33. A. F. Brilot, A. A. Korostelev, D. N. Ermolenko, N. Grigorieff, Structure of the ribosome with elongation factor $\mathrm{G}$ trapped in the pretranslocation state. Proc Natl Acad Sci US A 110, 20994-20999 (2013).

34. J. Chen et al., Coupling of mRNA Structure Rearrangement to Ribosome Movement during Bypassing of Non-coding Regions. Cell 163, 1267-1280 (2015).

35. A. Dashti et al., Retrieving functional pathways of biomolecules from single-particle snapshots. Nat Commun 11, 4734 (2020).

36. K. Luger, T. J. Rechsteiner, T. J. Richmond, Preparation of nucleosome core particle from recombinant histones. Methods Enzymol 304, 3-19 (1999).

37. A. Flaus, K. Luger, S. Tan, T. J. Richmond, Mapping nucleosome position at single basepair resolution by using site-directed hydroxyl radicals. Proc Natl Acad Sci U S A 93, 1370-1375 (1996).

38. C. A. Sarkar et al., Directed evolution of a $\mathrm{G}$ protein-coupled receptor for expression, stability, and binding selectivity. Proc Natl Acad Sci U S A 105, 14808-14813 (2008). 
39. G. Lebon, K. Bennett, A. Jazayeri, C. G. Tate, Thermostabilisation of an agonist-bound conformation of the human adenosine A(2A) receptor. J Mol Biol 409, 298-310 (2011).

40. T. Liu et al., Enhancing protein stability with extended disulfide bonds. Proc Natl Acad Sci U S A 113, 5910-5915 (2016).

41. M. Zavodszky et al., Disulfide bond effects on protein stability: designed variants of Cucurbita maxima trypsin inhibitor-V. Protein Sci 10, 149-160 (2001).

42. D. M. Rosenbaum et al., GPCR engineering yields high-resolution structural insights into beta2-adrenergic receptor function. Science 318, 1266-1273 (2007).

43. V. Cherezov et al., High-resolution crystal structure of an engineered human beta2adrenergic G protein-coupled receptor. Science 318, 1258-1265 (2007).

44. S. G. Rasmussen et al., Crystal structure of the human beta2 adrenergic G-proteincoupled receptor. Nature 450, 383-387 (2007).

45. H. Stark, GraFix: stabilization of fragile macromolecular complexes for single particle cryo-EM. Methods Enzymol 481, 109-126 (2010).

46. B. Kastner et al., GraFix: sample preparation for single-particle electron cryomicroscopy. Nat Methods 5, 53-55 (2008).

47. T. Nakane, D. Kimanius, E. Lindahl, S. H. Scheres, Characterisation of molecular motions in cryo-EM single-particle data by multi-body refinement in RELION. Elife 7 (2018).

48. A. Punjani, D. J. Fleet, 3D variability analysis: Resolving continuous flexibility and discrete heterogeneity from single particle cryo-EM. J Struct Biol 213, 107702 (2021).

49. S. H. Scheres, Processing of Structurally Heterogeneous Cryo-EM Data in RELION. Methods Enzymol 579, 125-157 (2016).

50. B. Chen, B. Shen, J. Frank, Particle migration analysis in iterative classification of cryoEM single-particle data. J Struct Biol 188, 267-273 (2014).

51. E. Lerner et al., Toward dynamic structural biology: Two decades of single-molecule Förster resonance energy transfer. Science 359, eaan1133 (2018).

52. H. Mazal, G. Haran, Single-molecule FRET methods to study the dynamics of proteins at work. Curr Opin Biomed Eng 12, 8-17 (2019).

53. G. Sirinakis, Y. Ren, Y. Gao, Z. Xi, Y. Zhang, Combined versatile high-resolution optical tweezers and single-molecule fluorescence microscopy. Rev Sci Instrum 83, 093708 (2012).

\section{Acknowledgments:}

We thank Prof. Yifan Cheng from UCSF for thorough discussions. We thank Prof. Abbas Ourmazd and Dr. Ghoncheh Mashayekhi from UW-Milwaukee for help on ManifoldEM. We are grateful to Kang Li, Dianli Zhao and Ceng Gao from the CryoEM facility for Marine Biology at QNLM for our cryo-EM data collection.

\section{Funding:}

National Key R\&D program of China, 2017YFA0504800 (QS)

National Key R\&D program of China, 2018YFC1406700 (QS)

National Natural Science Foundation of China, 31870743 (QS) 


\section{Author contributions:}

Conceptualization: QS

Methodology: XC, XS, LQ, GL, YL, JL

Investigation: XC, XS, ML, LL, TL, YQ, GL

Visualization: XC, XS, QS

Funding acquisition: QS

Project administration: QS

Supervision: QS

Writing - original draft: XC, XS, LL, QS

Writing - review \& editing: QS

Competing interests Authors declare no competing interests.

Data availability The cryo-EM density maps of ribosomes under all conditions (S1-S10) were deposited in Electron Microscopy Data Bank (EMDB) with the accession numbers from 31266 to 31275 , as listed in fig. S2B. The locally refined cryo-EM maps of the $30 S$ subunits from the unannealed (S1) and annealed (S5) ribosomes were also deposited in EMDB with the respective accession numbers of 31277 and 31279 . Cryo-EM density maps and models for all 50 states in free energy landscape analysis are available from the corresponding author by request. All other data is available in the main text or the supplementary materials.

Code availability The codes for local resolution analysis and modified ManifoldEM have been uploaded to GitHub: https://github.com/soothing35/cryoEM_annealling.

\section{Supplementary Materials:}

Materials and Methods

Supplementary Text

Figs. S1 to S13

Table S1

Movies S1 to S2 


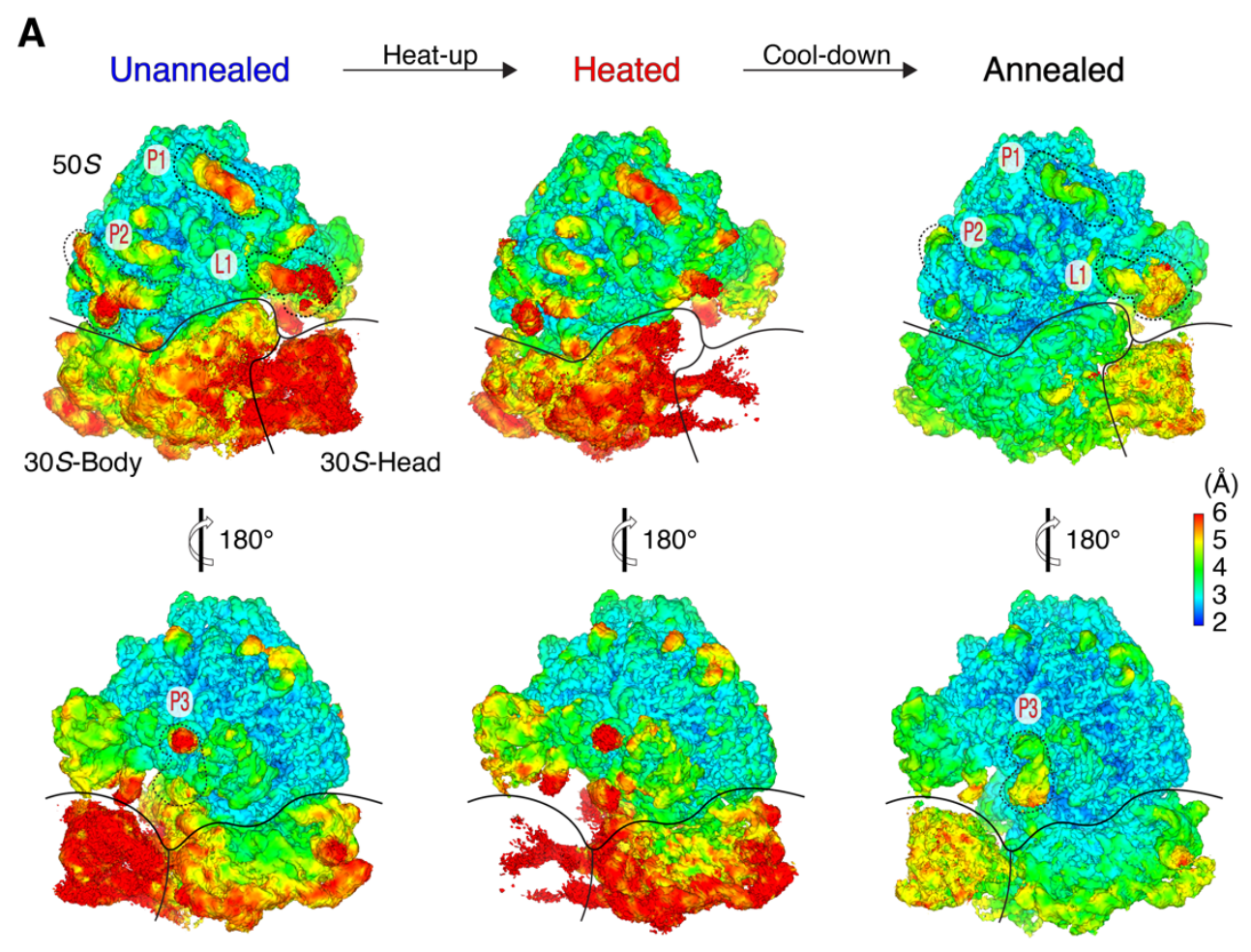

B

Fig. 1. Annealing improves local resolution of the $70 S$ ribosome. (A) Local resolution maps of the unannealed, heated, and annealed $70 S$ ribosomes. The $50 S$ subunit and the body and head domains of the $30 S$ subunit are labeled. L1 stalk (L1) and other three sub-regions (P1, P2, and P3) on the periphery of the $50 \mathrm{~S}$ subunit in the unannealed and annealed $70 \mathrm{~S}$ ribosomes are marked for resolution comparison. (B) Local resolution comparison of the unannealed, heated, and annealed $70 S$ ribosomes. 

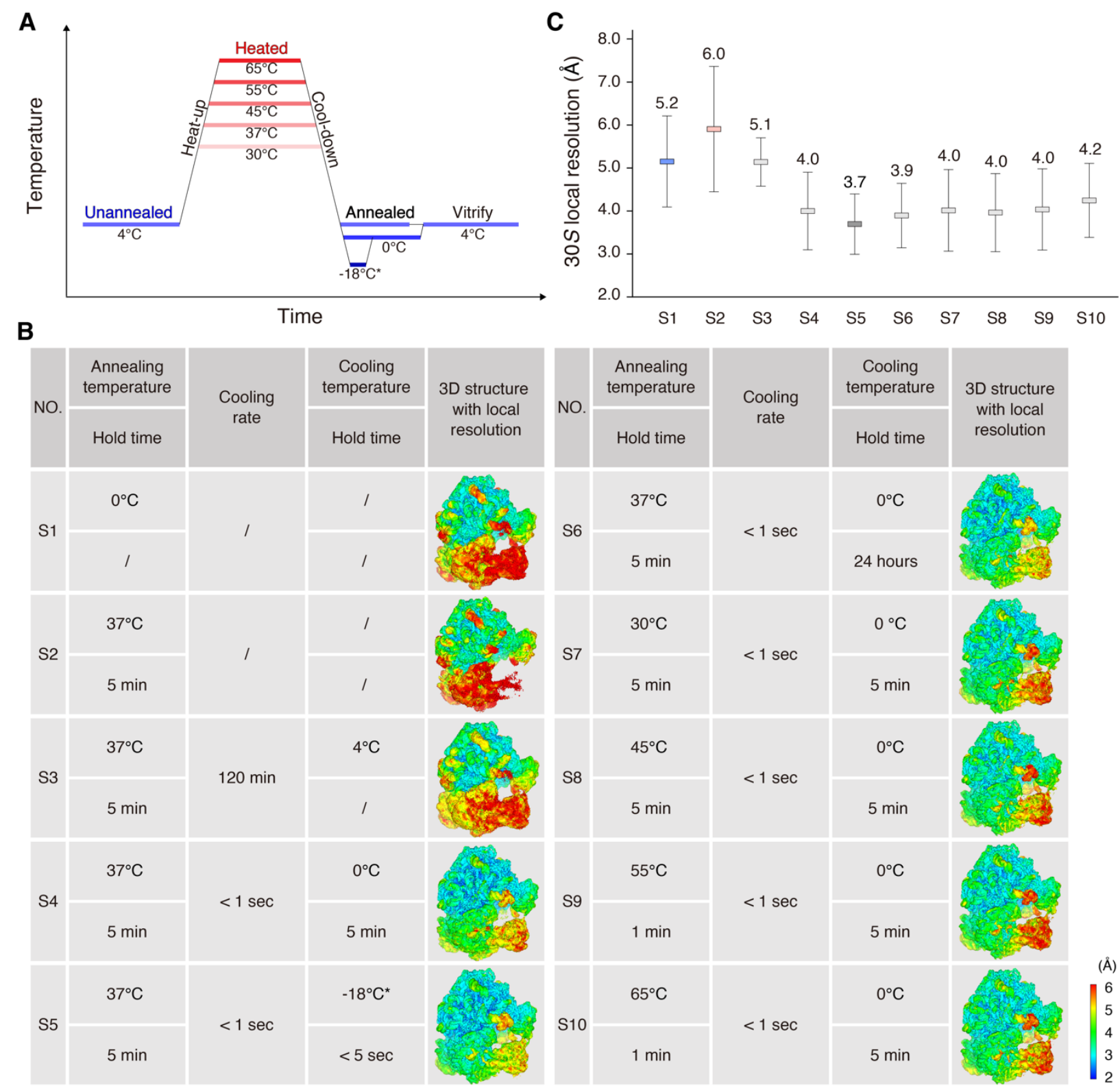

Fig. 2. Systematic screening on annealing conditions. (A) Schematic for annealing of the 70S ribosome. The unannealed, heated, and annealed stages are labeled on the diagram. (B) Detailed annealing conditions on the $70 S$ ribosomes and the respective 3D local resolution maps. We used sample names S1-S10 in (C) and other figures. Regarding S5, we briefly $(<5 \mathrm{~s})$ immersed the $70 S$ ribosome into a mixture of salt, ice, and water pre-cooled to $-18^{\circ} \mathrm{C}$, and then transferred the samples to a mixture of ice and water at $0^{\circ} \mathrm{C}$ before freezing. $(\mathbf{C})$ Local resolution comparison of $30 S$ subunits among various annealing conditions. 
A

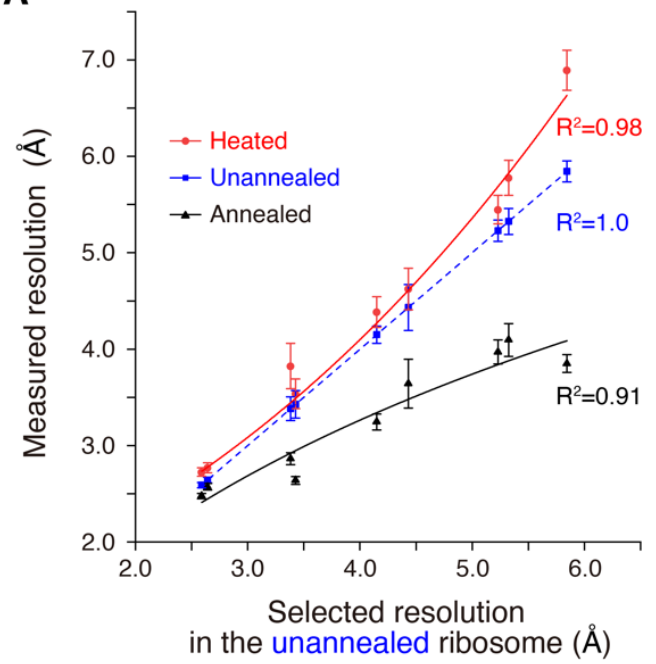

B

C

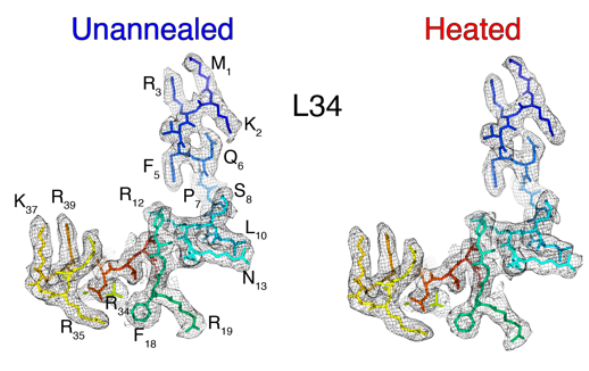

H52
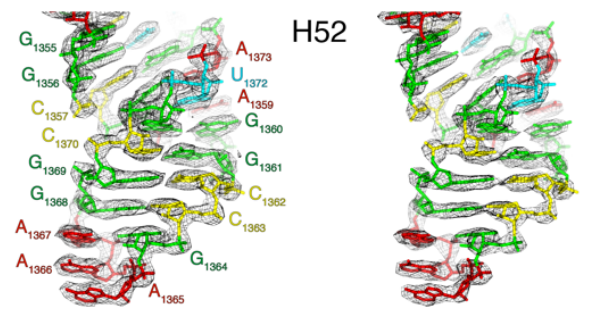

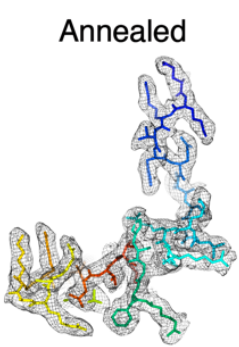

F

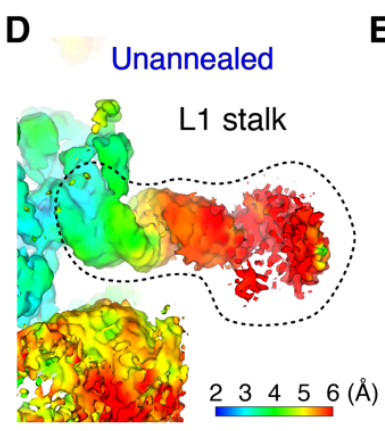

E

Annealed
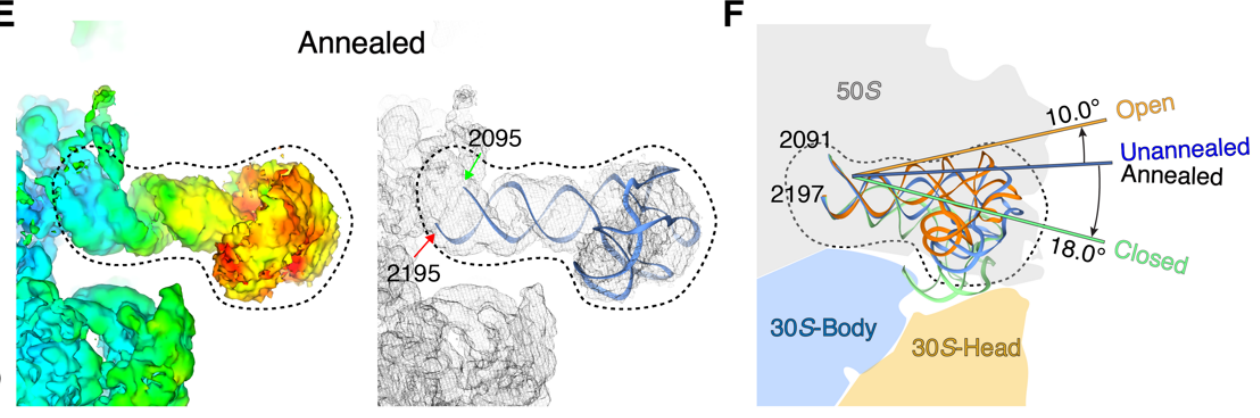

Fig. 3. Annealing stabilizes flexible regions of the $70 S$ ribosome. (A) The effects of heating and annealing on regions with different local resolutions. We selected regions with different local resolutions from 2.5-6.0 $\AA$ in the unannealed $70 S$ ribosome, and measured local resolutions in the corresponding regions in the heated and annealed $70 S$ ribosomes. (B) and (C) Cryo-EM maps and fitted atomic models for L34 ribosomal protein and H52 helix in 23S rRNA under unannealed, heated, and annealed conditions. (D) Local resolution map of the L1 stalk in the unannealed ribosome. The position of the L1 stalk is shown in Fig. 1A. (E) Local resolution map of the L1 stalk in the annealed ribosome and fitted atomic model. (F) Intermediate states of the L1 stalk in the unannealed and annealed ribosomes, with respect to the known open and closed states. Atomic models of the L1 stalk are as follows: open (gold), intermediate (blue), and closed (green) states. 
A

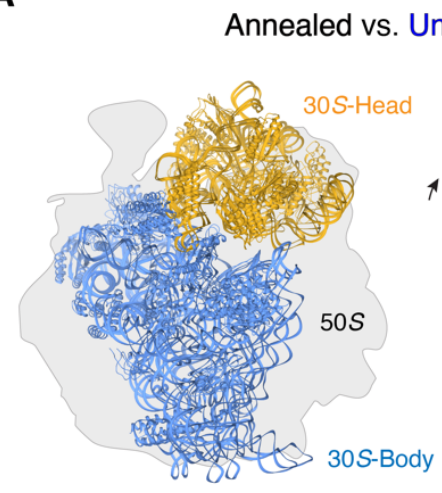

Annealed vs. Unannealed

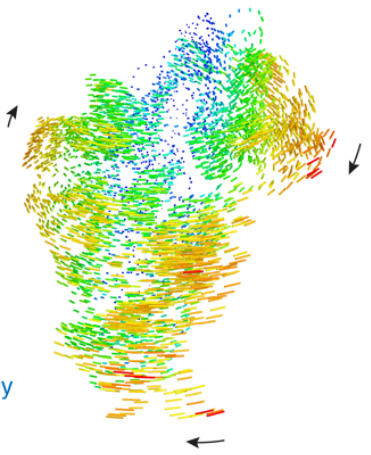

C

Unannealed vs. Rotated/Nonrotated
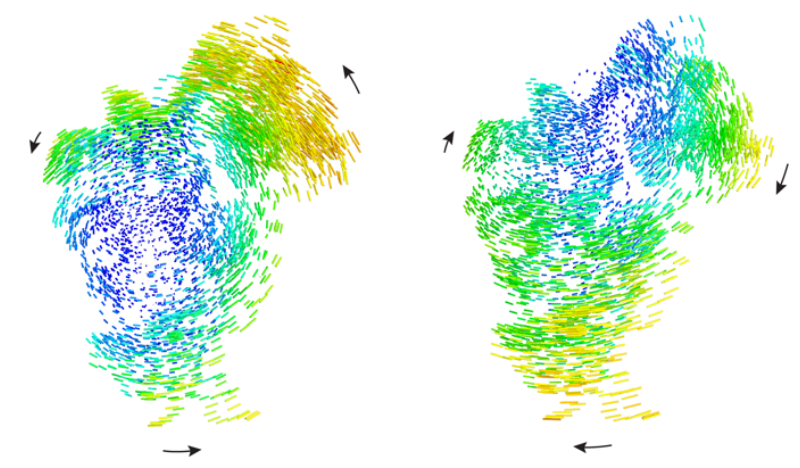

B

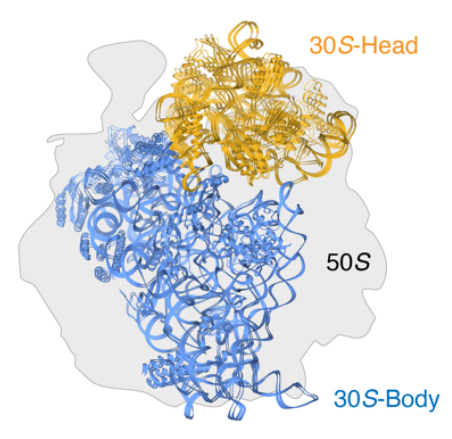

Heated vs. Unannealed

D
Annealed vs. Nonrotated

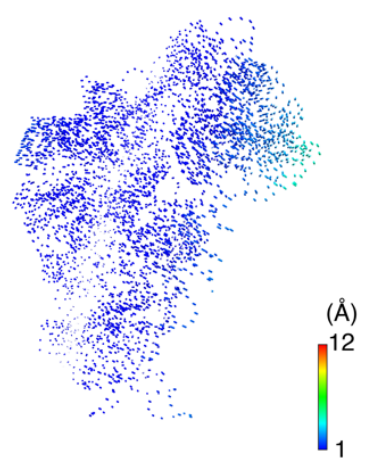

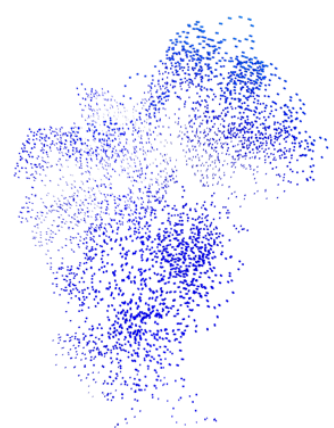

E

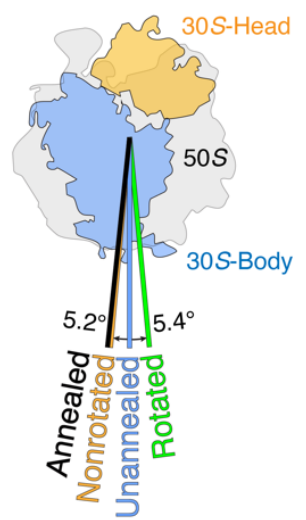

Fig. 4. Annealing renders the $70 S$ ribosome into a nonrotated state. (A) Rotational comparison of $30 S$ subunits between the annealed and unannealed ribosomes. Left: We aligned the $50 S$ subunits in the annealed and unannealed ribosomes as a reference, and atomic models for the $30 S$ subunits are shown. Right: Difference vectors between phosphorous and C $\alpha$ atoms in the $30 S$ subunits, with arrows indicating the direction of the change. (B) Rotational comparison of $30 S$ subunits between the heated and unannealed ribosomes. (C) Rotational comparison of $30 S$ subunit in the unannealed ribosome against the known fully rotated (PDB ID 4V9D, Left) and nonrotated (PDB ID 4V7C, Right) states. (D) Rotational comparison of $30 S$ subunit in the annealed ribosome against the nonrotated state. (E) Summary of rotation angles of $30 S$ subunit among the unannealed and annealed ribosomes, and the rotated and nonrotated states. 
A

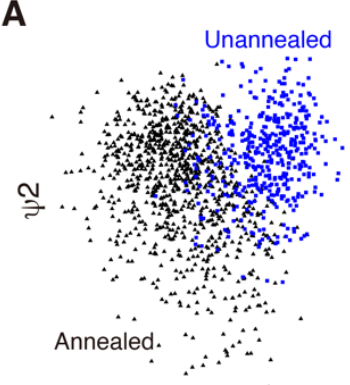

$\psi 1$
B

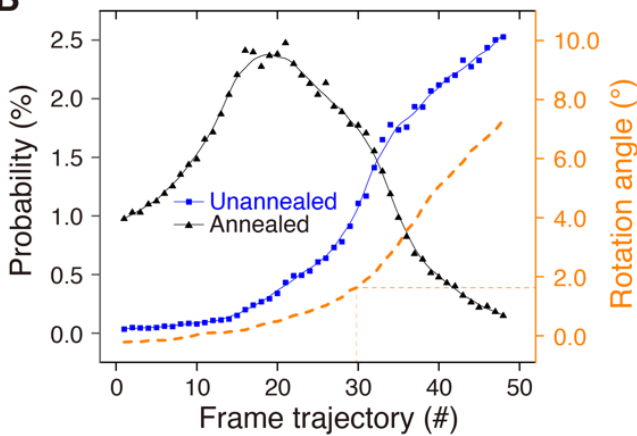

E
D

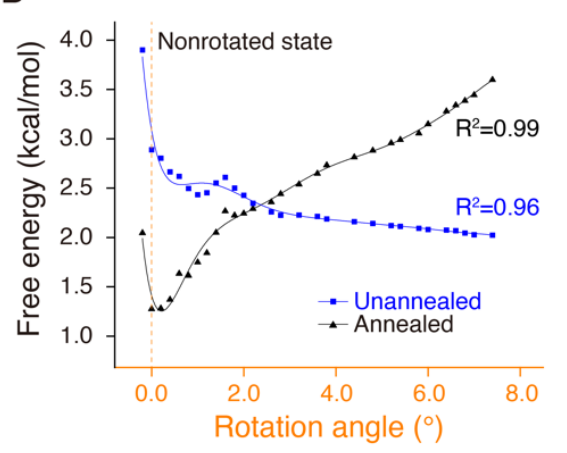

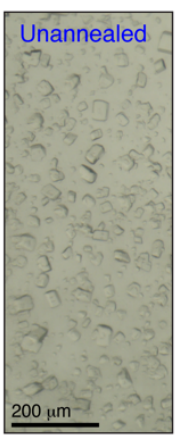

C

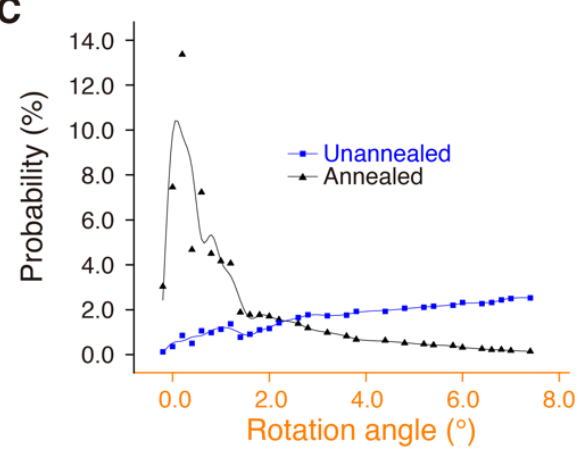

F
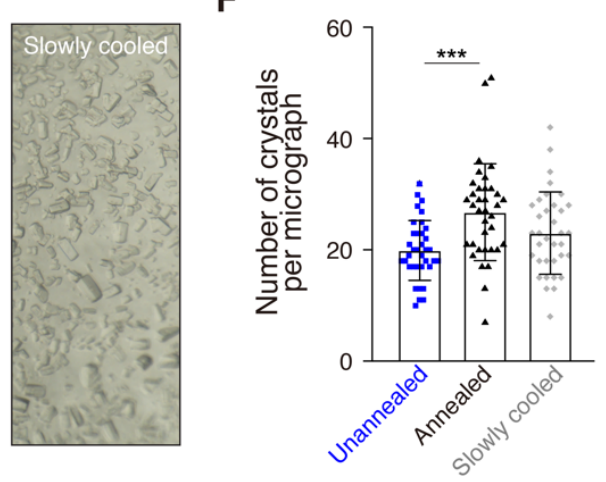

政

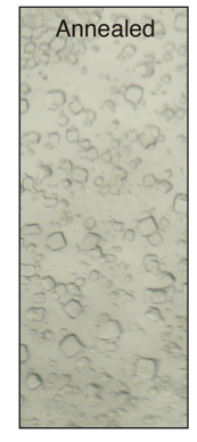

Fig. 5. Annealing minimizes the free energy of the $70 S$ ribosome. (A) Initial manifold snapshots of the $70 S$ ribosome in one projection direction (points colored in accordance with the unannealed and annealed subsets). The projection direction is approximately orthogonal to the interface between the $50 S$ and $30 S$ subunits. (B) Particle distribution of unannealed and annealed ribosomes along the frame trajectory. We reconstructed the $3 \mathrm{D}$ structure at each frame and calculated the rotation angle of the $30 S$ subunit with respect to the nonrotated state. (C) Particle distribution of the unannealed and annealed ribosomes along the rotation angle. We recalculated particle numbers in accordance with the rotation angle at intervals of $0.2^{\circ}$ and used a moving average to smooth data variation. (D) Free-energy distribution of unannealed and annealed ribosomes along the rotation angle. We calculated the energy from the fitted curve in $(\mathbf{C})$. (E) and (F) Growth rate comparison of ribosome crystals: unannealed, annealed, and slowly cooled. We show three representative images for ribosome crystals under three conditions $(\mathbf{E})$, and a statistical analysis on the numbers of crystals per micrograph (F). We randomly selected 36 images per condition and performed analysis of variance for comparison. 


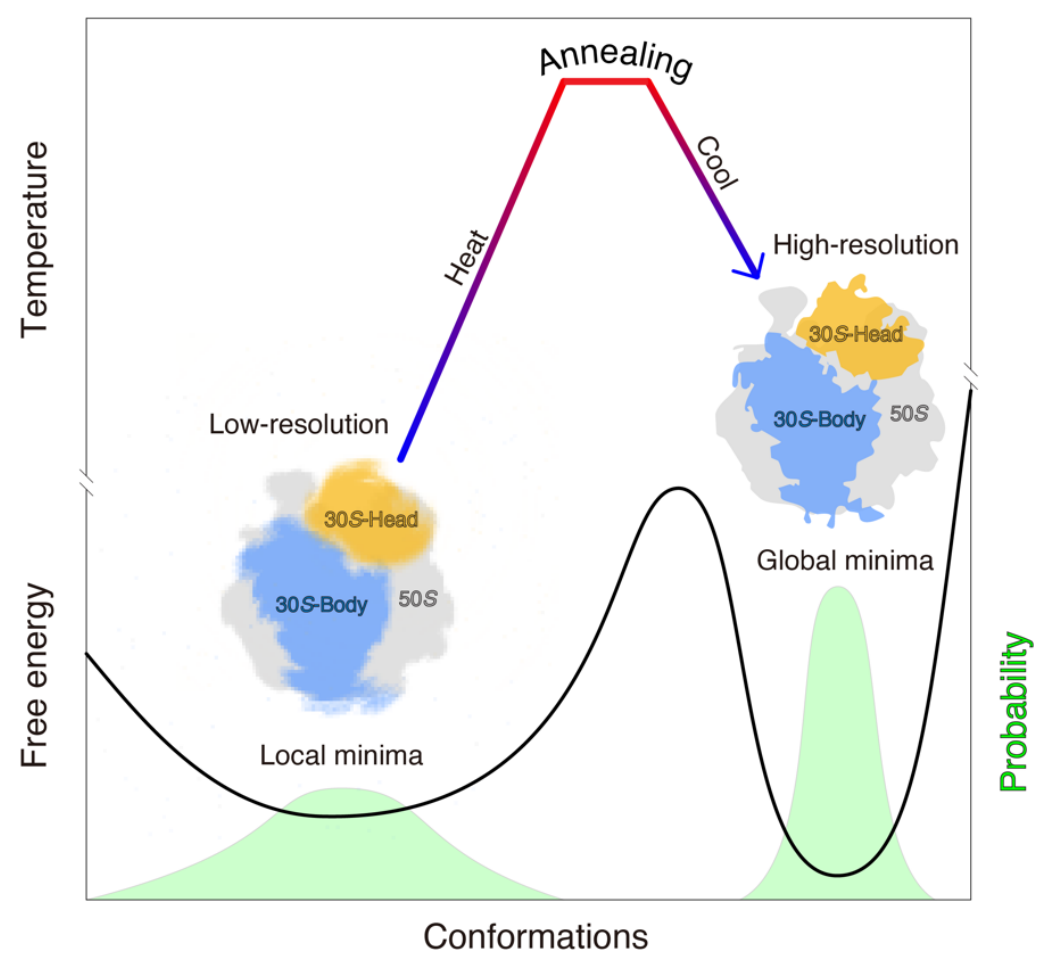

Fig. 6. Model illustrating that annealing can synchronize a ribosome into a minimumenergy state with improved local resolution. Both the free-energy curve (solid line) and particle distribution probability (light green peaks) are shown. 


\section{Supplementary Materials for}

\section{Annealing synchronizes the $70 S$ ribosome into a minimum-energy conformation}

Authors: Xiaofeng $\mathrm{Chu}^{1,2 \dagger}$, Xin $\mathrm{Su}^{1 \dagger}$, Mingdong $\mathrm{Liu}^{1,3}, \mathrm{Li} \mathrm{Li}^{4}$, Tianhao $\mathrm{Li}^{1,3}$, Yicheng Qin ${ }^{1}$,

Affiliations:

9 iHuman Institute and School of Life Science and Technology, ShanghaiTech University,

10 Shanghai 201210, China.

$11{ }^{2}$ Laboratory for Marine Biology and Biotechnology, Qingdao National Laboratory for Marine

12 Science and Technology, Qingdao 266237, China.

13 '3niversity of Chinese Academy of Sciences, Beijing 100049, China.

$14{ }^{4}$ State Key Laboratory of Genetic Engineering, School of Life Sciences, Zhongshan Hospital, 15 Fudan University, Shanghai, 200438.

16 These authors contributed equally: Xiaofeng $\mathrm{Chu}, \mathrm{Xin} \mathrm{Su}$

$17{ }^{*}$ Corresponding author. Email: Qing-Tao Shen (shenqt@shanghaitech.edu.cn)

18

19 This PDF file includes:

20 Materials and Methods

21 Supplementary Text

22 Figs. S1 to S13

23 Table S1

24 Captions for Movies S1 to S2 
Materials and Methods

\section{Purification and assembly of the apo-state $70 S$ ribosome}

$70 S$ ribosomes were extracted from Escherichia coli $\mathrm{KC} 6 / \Delta \mathrm{smpB} / \Delta \mathrm{ssrA} / \mathrm{S} 1:: \mathrm{S} 1-8 \mathrm{xH}$ is

\section{Cryo-EM sample preparation}

The frozen $70 S$ ribosomes were melted on ice and then diluted to a final concentration of $700 \mathrm{nM}$ in HEPES (5 mM, pH 7.5) buffer containing $50 \mathrm{mM} \mathrm{KCl}, 10 \mathrm{mM} \mathrm{NH} 4 \mathrm{Cl}, 10 \mathrm{mM}$ $\mathrm{Mg}(\mathrm{OAc})_{2}$, and $2 \mathrm{mM}$ 2-Mercaptoethanol. To ensure fast heat exchange, no more than $20 \mu \mathrm{L}$ of the diluted ribosomes were pipetted into one PCR tube. All the samples were subjected to various treatments as listed in Fig. 2B, before vitrification.

For the annealed treatments (S4-S10), the diluted ribosomes were placed on ice for $5 \mathrm{~min}$ and then transferred to a water bath, with preset temperatures ranging from $30^{\circ} \mathrm{C}$ to $65^{\circ} \mathrm{C}$. After a 1- or 5-min hold time, the heated samples were immediately immersed into a mixture of salt, ice, and water (measured temperature: $-18^{\circ} \mathrm{C}$ ) for $<5 \mathrm{~s}$; and then to a mixture of ice and water (measured temperature: $-0^{\circ} \mathrm{C}$ ), or directly immersed into a mixture of ice and water for either 5 min or $24 \mathrm{~h}$. As a control, the unannealed ribosome (S1) was simply immersed in a mixture of ice and water for $5 \mathrm{~min}$. For the slowly annealed sample (S3), the diluted ribosome in the PCR tube was placed in a PCR machine (Bio-Rad, USA). The temperature decreased gradually from an initial value of $37^{\circ} \mathrm{C}$ to a final value of $4^{\circ} \mathrm{C}$ over $2 \mathrm{~h}$ at a cooling rate of $0.275^{\circ} \mathrm{C} / \mathrm{min}$.

After treatments, all the unannealed and annealed samples in the PCR tubes were immediately placed in a Vitrobot chamber set at the minimum temperature of $4{ }^{\circ} \mathrm{C}$ and a humidity of $100 \%$ (Mark IV, Thermo Fisher Scientific, USA). A gold-supported holey carbon grid was glow discharged and pre-cooled in the Vitrobot chamber for 2 min, and $4 \mu \mathrm{L}$ of solution was applied onto the grid. The grid was immediately plunged into liquid ethane and stored under liquid nitrogen for future cryo-EM imaging. 


\section{Cryo-EM sample preparation with a mist umbrella}

In temperature-resolved cryo-EM, sudden exposure of high-temperature mist $\left(\sim 37^{\circ} \mathrm{C}\right)$

\section{Cryo-EM data processing and 3D reconstruction} from a Vitrobot chamber to cooler ambient temperatures $\left(\sim 20^{\circ} \mathrm{C}\right)$ causes substantial ice contamination. To minimize ice contamination under various temperatures, especially at high temperatures, a mist umbrella was designed. The mist umbrella was made of a piece of round filter paper $(40-\mathrm{mm}$ diameter) with a precut square hole $(9.0 \mathrm{~mm} \times 3.6 \mathrm{~mm})$ prepared via a home-made device (fig. S7). The peripheral belt of the mist umbrella was pre-treated with lowadhesive glue. Before lifting the Vitrobot tweezer into the chamber, the mist umbrella was clamped to the tweezer via the precut hole. Over the course of lifting the grid to the chamber, the mist umbrella fell off the tweezer yet glued to the bottom surface of the chamber. After blotting, the shutter of the Vitrobot chamber was immediately opened and the grid passed through the precut hole into liquid ethane. The high-temperature mist in the chamber was blocked by the mist umbrella, which prevents the exposure of high-temperature mist to the cool ambient. When the liquid nitrogen reservoir and cryogenic cup dropped away from the chamber, the tweezer reattached the mist umbrella to shield the grids (movie S1). During grid transfer from liquid ethane to liquid nitrogen, one can readily remove the mist umbrella from the Vitrobot tweezer along the tear line.

The mist umbrella was used for the heated ribosome (S2) as an intermediate state. Diluted ribosomes were incubated in a water bath at $37^{\circ} \mathrm{C}$ for $5 \mathrm{~min}$, and then transferred into the Vitrobot chamber at a temperature of $37^{\circ} \mathrm{C}$ and a humidity of $100 \%$. The grid was placed in the chamber for at least $100 \mathrm{~s}$ to ensure complete heat exchange, and then $5 \mu \mathrm{L}$ of solution was applied onto the grid via pre-heated tips. The grids were blotted with a $1 \mathrm{~s}$ blotting time and force level of 2, immediately plunged into liquid ethane, and stored under liquid nitrogen for future cryo-EM imaging.

\section{Cryo-EM data collection}

Cryo-EM grids were examined in a low-dose mode on a Talos L120C TEM (Thermo Fisher Scientific, USA) for screening. Data collection on high-quality grids in all conditions was performed with the same Titan Krios $\mathrm{G}^{3 \mathrm{i}}$ microscope (Thermo Fisher Scientific, USA) equipped with a K3 BioQuantum direct electron detector (Gatan, USA). Special care was taken to perform a coma-free alignment on the microscope.

Movies were collected via FEI EPU (3) automated data collection software at a total dose of $60 \mathrm{e}^{-} / \AA^{2}$ fractionated over 50 frames in a defocus range of -1 to $-1.5 \mu \mathrm{m}$. A super-resolution mode was used with a final pixel size at $0.53 \AA$. The numbers of the collected movies under different conditions are listed in fig. S2B and table S1.

For optimal structural comparison, the same image processing strategy was performed on ribosomes under all conditions with cryoSPARC v.3.1.0 (4); fig. S2 shows a detailed workflow. Specifically, raw movie stacks were aligned and summed in accordance with dose weighting with MotionCor2.1 (5). CTF parameters of the summed micrographs were determined with CTFFIND-4 (6). Micrographs with maximum resolution estimates of at least $5 \AA$ were imported into cryoSPARC. Automatic particle picking was performed on the selected micrographs, and 
particle sets were created and subjected to reference-free 2D classifications. Obvious junk was excluded from the particle set. Six reference structures-including $2 \times 70 S$ ribosome (PDB ID $5 \mathrm{MDZ}$ ), $2 \times 50 S$ subunit (derived from PDB ID 5MDZ), and $2 \times 30 S$ subunit (derived from PDB ID 5MDZ) — were low-pass filtered to $40 \AA$ and utilized for heterogeneous refinement. Only particles in the integral $70 S$ ribosome with sufficient signal on the $30 S$ subunit were collected. Two hundred thousand particles were randomly selected from the new particle set for each condition, and per-particle refinement of CTF parameters was conducted. Particles with local CTF parameters were subjected to homogeneous refinement. All the final reconstructions were filtered and sharpened by a cryoSPARC post-processing session and the resolutions were determined by gold-standard FSC 0.143 .

\section{Model building and structural analysis}

The crystal structure of the $70 S$ ribosome, with an empty A site (PDB ID 5MDZ) (7), from $E$. coli was chosen as the initial template; and mRNA and fMet-tRNA were removed before fitting into the apo-state $70 S$ ribosomes. The $50 S$ and $30 S$ subunits of the modified crystal structure were separately fit into the $70 \mathrm{~S}$ ribosomes under various conditions via rigid-body docking, and the fits were ideal except on the L1 stalk. A flexible fitting of the L1 stalk was performed with Rosetta 2018.33.60351 (8). The atomic model was further optimized for improved local density fitting with Coot 0.8.9.1 (9) and real-space refinement with PHENIX 1.17.1 (10).

Structures of the unannealed, heated, and annealed $70 S$ ribosomes were aligned using their $50 S$ subunits as a reference. The conformational change of the $30 S$ subunits was analyzed in Pymol 2.4.0 as described elsewhere $(11,12)$. Difference vectors between the phosphorous and $\mathrm{C} \alpha$ atoms in the unannealed, heated, and annealed $30 S$ subunits were calculated via the modified script (https://github.com/soothing35/cryoEM annealling/Pymol scripts), and rotation angles between the $30 \mathrm{~S}$ subunits were measured with the Pymol script 'RotationAxis.py' (https://pymolwiki.org/index.php/RotationAxis).

Other structural analysis, such as structural superimposition, were fulfilled with UCSF Chimera $1.16(13)$.

\section{Local refinement on the $30 S$ subunit}

144

Local refinement on the $30 S$ subunit was also performed with cryoSPARC (4). Based on the docked atomic model, a mask for the $50 S$ subunit was created and applied to subtract the corresponding densities in all of the particles. Local refinement was conducted on the $30 \mathrm{~S}$ subunit density with rotations and shifts re-centered every iteration. A new function in cryoSPARC termed "using pose/shift gaussian prior during alignment" was also used for improved reconstruction. The final reconstruction was filtered and sharpened by a cryoSPARC post-processing session and the resolutions were determined by gold-standard FSC 0.143.

\section{Local resolution quantification}

To compare local resolutions of the $30 S$ subunits and other sub-regions under various conditions, local resolution quantification was calculated via averaging values of the local 
resolution in selected regions (fig. S4). Specifically, local resolutions on the entire $70 S$ ribosomes under various conditions were first estimated as per local resolution estimation with cryoSPARC (14). Then the regions within a certain local resolution range or within the same functional domain were selected as the targets. After atomic model building, a mask corresponding to the targeted region was created with the extension of 2 pixels, and the same mask strategy was applied to the targeted regions from other structures. The resulting sub-regions were adjusted to the same volume among different structures, and the values of the local resolution within the read via our

Python script

162 (https://github.com/soothing35/cryoEM_annealling/locres_points). The numbers of local resolution values in the range of 2.0-2.1 $\AA, 2.1-2.2 \AA$, and so on were counted in different subregions. The respective means and standard deviations were calculated for each sub-region and compared with each other.

\section{Free-energy landscape analysis}

Free energy is inversely proportional to particle distribution, as described by the Boltzmann distribution (15). The particle distribution of $70 S$ ribosomes under various conditions was calculated with ManifoldEM (https://github.com/GMashayekhi/ManifoldEM Matlab) as described elsewhere $(16,17)$. Specifically, 100,000 particles were randomly selected from screened particle sets for each condition to sample the entire conformational trajectory. Particles files, including both .star and .mrcs, were selected after auto-refinement with RELION 3.0.8 (18). To ensure accurate alignment of structures from two different conditions, their particle files were merged together via appending one to the other with different labels, and the merged particle files were analyzed with ManifoldEM for further analysis.

In ManifoldEM, merged particles were classified in accordance with the pre-determined Euler angels, and particle images were deemed as dots in high-dimensional space. Clouds of dots in high-dimensional space were eigen-decomposed and embedded into low-dimensional space with a diffusion map algorithm, where the kernel was defocus-tolerant. The first five eigenvectors were selected and the conformational changes along each eigen-function were extracted into 2D movies by nonlinear Laplacian spectral analysis (NLSA), also termed non-linear singular value decomposition (SVD). Considering that the inter-subunit rotation between the $50 S$ and $30 S$ subunits was considered to be the primary conformational change, movies with such conformational changes along each eigen-function were chosen manually. Particles involved in the conformational changes were further classified into 50 classes along the conformational trajectory.

For all 50 classes, 3D volumes were reconstructed via back-projection in RELION 3.0.8. Atomic models for the $50 S$ and $30 S$ subunits were separately docked in each volume via rigidbody docking. All structures were aligned to the nonrotated state (PDB ID 4V9D) with the 50S subunit as a reference. The rotation angle of the $30 S$ subunit in each class was calculated with respect to the $30 S$ subunit in the nonrotated state. Based on the labels of different conditions, particles in each class were separated. The particle distribution within the same condition was recalculated and the respective free energy was calculated based on the Boltzmann distribution. 


\section{Ribosome crystallization}

E. coli $70 S$ ribosomes were readily crystalized when their native ribosomal protein L9 was replaced with Thermus thermophilus ribosomal protein L9 (19). E. coli L9-free $70 S$ ribosomes were expressed in $E$. coli $\mathrm{KC} 6 / \Delta \mathrm{smpB} / \Delta \mathrm{ssrA} / \mathrm{S} 1:: \mathrm{S} 1-8 x H i s /$ EcoL9(40)FRT strain and purified as described above. $T$. th ribosomal protein L9 was expressed in E. coli and obtained through multiple purification steps, including heating at $70^{\circ} \mathrm{C}$ to denature $E$. coli endogenous proteins, anion exchange chromatography via Mono $S$ HR 16/10, and gel filtration chromatography in HiLoad 26/600 Superdex 75pg. Subsequently, the purified $T$. th L9 protein were concentrated and stored in Tris- $\mathrm{HCl}(10 \mathrm{mM}, \mathrm{pH} 7.4)$ buffer containing $50 \mathrm{mM} \mathrm{KCl}$ and 1 mM 2-Mercaptoethanol.

E. coli $\mathrm{L} 9$-free $70 S$ ribosome at a final concentration of $4 \mu \mathrm{M}$ was incubated with $8 \mu \mathrm{M}$ T. th L9 on ice bath in the HEPES buffer. The mixtures were split into three portions and each portion was subjected to various annealing treatments. One portion of proteins was fast immersed into the mixture of salt, ice and water after heating to $37^{\circ} \mathrm{C}$, and then placed on ice bath (depicted as the annealed state); one portion of proteins was heated to $37^{\circ} \mathrm{C}$ and then slowly cooled down to $4^{\circ} \mathrm{C}$ over a period of 2 hours (depicted as the slowly cooled state); the third was kept on ice bath as the control.

The same crystallization conditions were applied to all annealing treated samples. Briefly, $3 \mu \mathrm{L}$ of sample and equal volume of precipitation $(2.9 \%(\mathrm{w} / \mathrm{v})$ PEG 20000, $100 \mathrm{mM}$ Tris-HCl, pH 7.4, 10\% (v/v) MPD, $160 \mathrm{mM}$ L-Arginine-HCl, and $0.5 \mathrm{mM}$ 2-Mercaptoethanol) were mixed and crystallized in a 24-well sitting drop plate containing $500 \mu \mathrm{L}$ of reservoir at $4^{\circ} \mathrm{C}$ for 14 days. For each condition, 36 images from seven drops were randomly captured, and crystals larger than $20 \times 20 \times 40 \mu \mathrm{m}^{3}$ were counted. The statistical analysis was fulfilled in GraphPad Prism 8.0. 


\section{Supplementary Text:}

\section{Structural comparison of $70 S$ ribosomes under various conditions}

1

In our work, $70 \mathrm{~S}$ ribosomes under various conditions were resolved via single-particle cryo-EM. Corresponding structural comparisons indicated that annealing could synchronize $70 \mathrm{~S}$ ribosomes, especially flexible regions, into homogenous states with improved local resolution. To objectively compare $70 \mathrm{~S}$ ribosomes, we tested reconstruction as follows:

1) Same batch of ribosomes. $70 \mathrm{~S}$ ribosomes were aliquoted and stored at $-80^{\circ} \mathrm{C}$ and unused ribosomes were discarded after thawing.

2) Same data collection conditions. The same microscope (Titan Krios $\mathrm{G}^{3 i}$ ) with the same camera (Gatan K3 Bioquantum) was used for all data collections. Total electron dose, defocus, and other pertinent values were set to be the same.

3) Same data processing pipeline. The procedure as described in fig. S2A was consistently applied to all $70 S$ ribosomes under various conditions.

4) Same data screening strategy. Only obvious junk and disassembled ribosomes were removed from the dataset. In most datasets, $82.3 \%$ to $93.7 \%$ of the particles were selected after 2D and 3D classifications. Three exceptions were the heated ribosome (S2) and annealed ribosomes ( $\mathrm{S} 9$ and $\mathrm{S} 10$ ). Ribosomes ( $\mathrm{S} 9$ and $\mathrm{S} 10$ ) were heated to $55^{\circ} \mathrm{C}$ and $65^{\circ} \mathrm{C}$, respectively (closer to the $\sim 72^{\circ} \mathrm{C}$ melting temperature of bacterial ribosome), for $5 \mathrm{~min}$; these $70 \mathrm{~S}$ ribosomes readily fell apart. Regarding S2, ribosomes were vitrified at $37^{\circ} \mathrm{C}$ as the heated state. Compared with ribosomes under other conditions, more unsatisfactory particles $(46.9 \%)$ were excluded from the final reconstruction, but the local resolution of $30 S$ subunit was still inferior.

5) Same number of particles $(200,000)$ for the final reconstruction, for comparison.

6) Repeating experiments. Repeats were performed on three representative conditions, including the unannealed (S1), heated (S2), and annealed (S5) ribosomes. A new batch of $70 \mathrm{~S}$ ribosomes was used and reconstructed. There was no obvious structural difference between two parallel experiments.

In summary, the structural differences should be attributable to differences in treatments. 

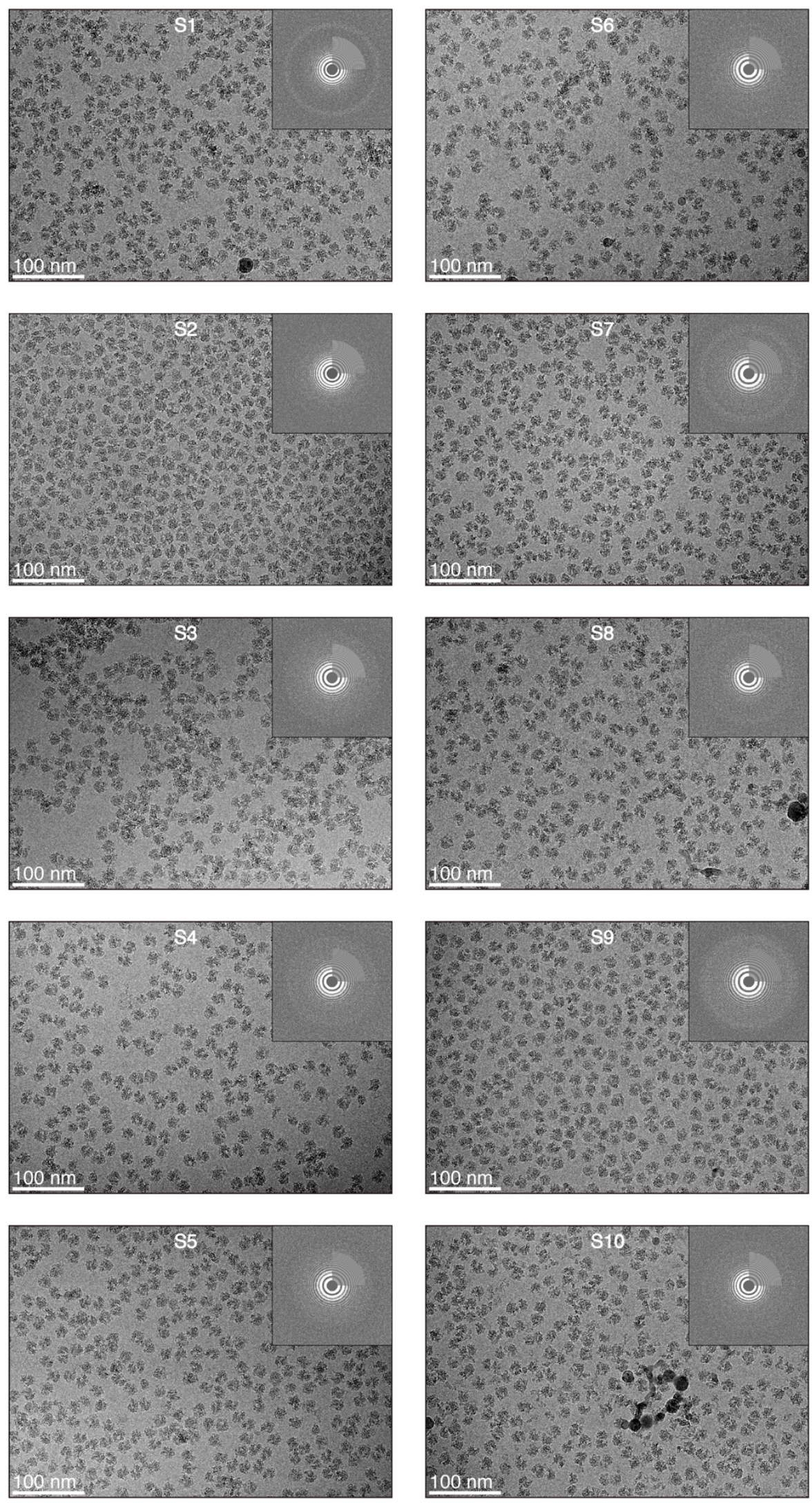
bioRxiv preprint doi: https://doi.org/10.1101/2021.06.25.447849; this version posted June 25, 2021. The copyright holder for this preprint (which was not certified by peer review) is the author/funder. All rights reserved. No reuse allowed without permission.

255 Fig. S1. Typical cryo-EM images under various conditions and their respective power 256 spectra

257 Fig. 2B lists detailed conditions for samples S1-S10.

258

259 
A

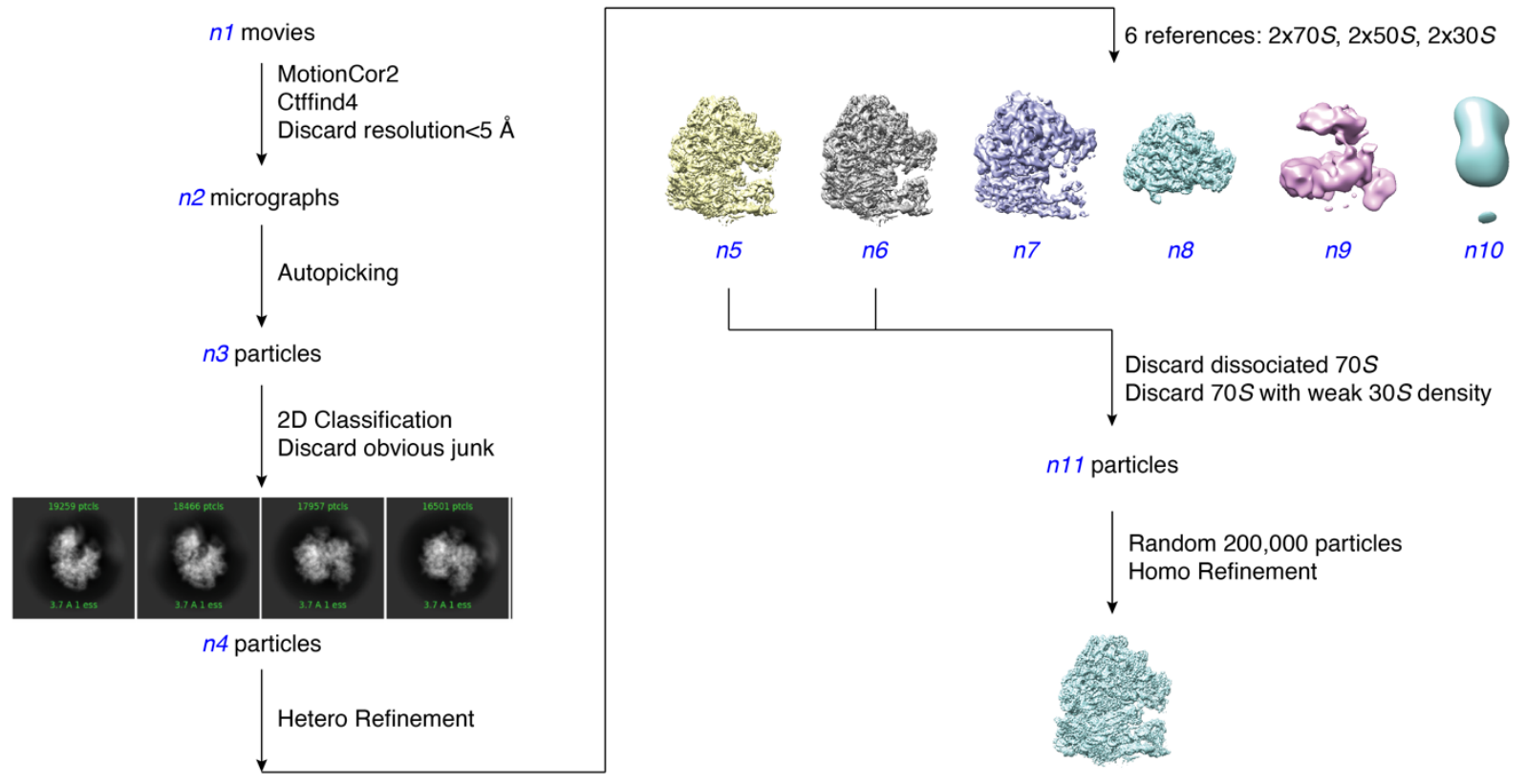

B

261 Fig. S2. 3D reconstructions of $70 S$ ribosomes under various conditions

262 (A) The same flow chart was used for 3D reconstruction of $70 S$ ribosomes under various 263 conditions. (B) Detailed numbers from $n 1-n 11$ in (A) and the corresponding deposited EMDB 264 IDs are listed. 
S1

GSFSC Resolution: $2.61 \AA$

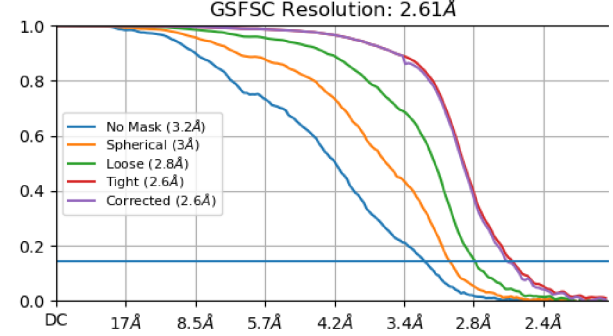

S2

GSFSC Resolution: $2.69 \AA$

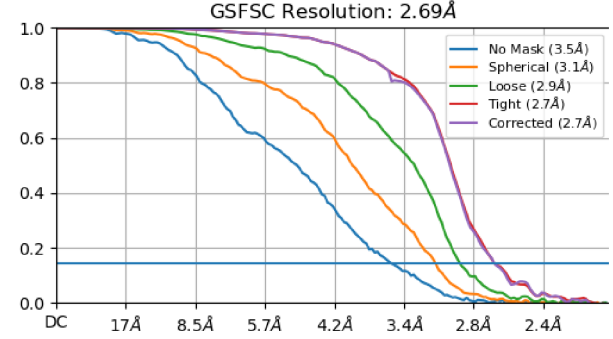

S3

GSFSC Resolution: $2.42 \AA$

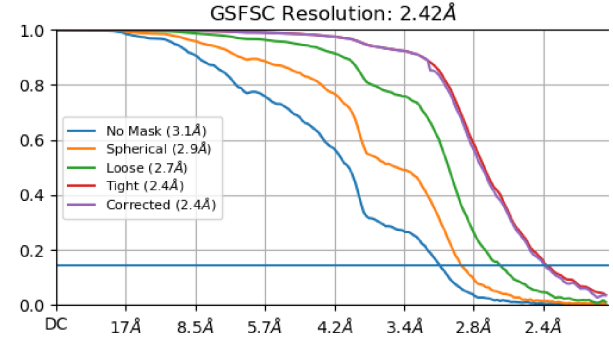

S4

GSFSC Resolution: $2.58 \AA$

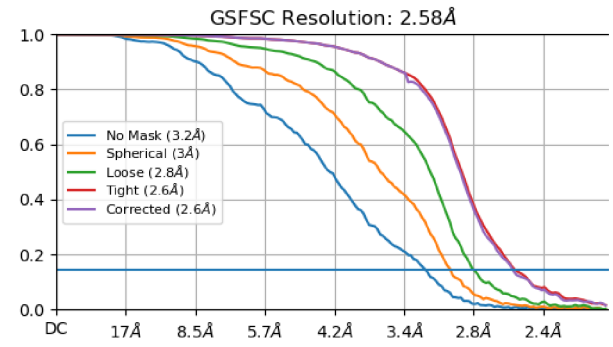

S5

GSFSC Resolution: $2.41 \AA$

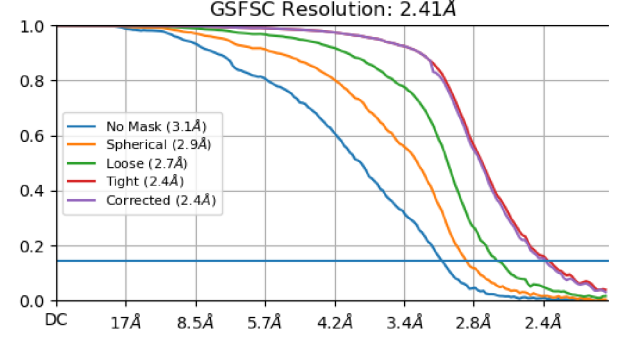

S6

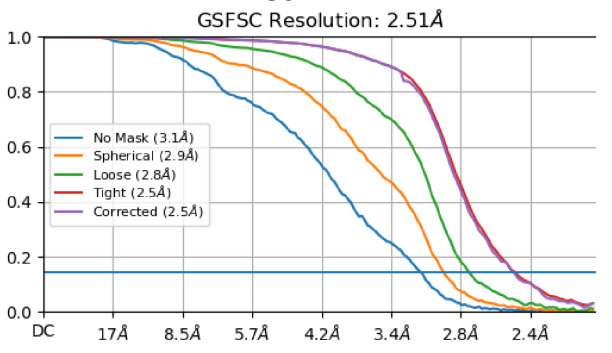

S7

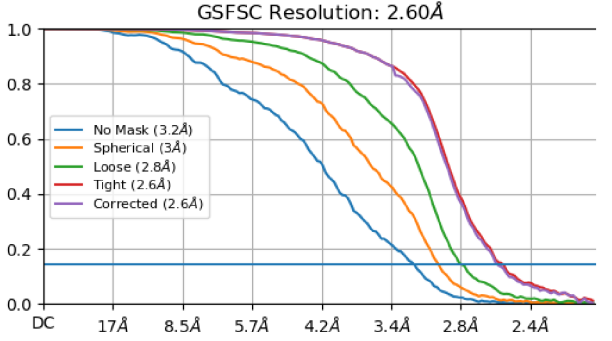

S8

GSFSC Resolution: $2.62 \AA$

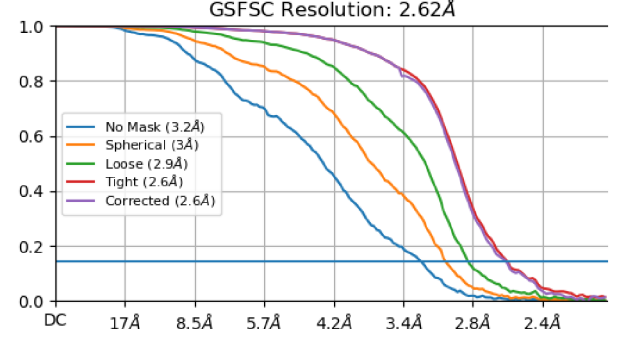

S9

GSFSC Resolution: $2.51 \AA$

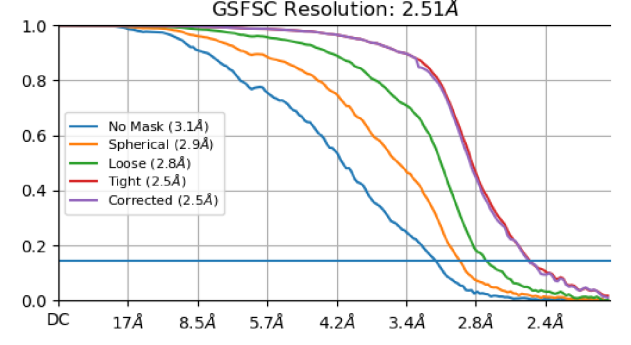

S10

GSFSC Resolution: $2.41 \AA$

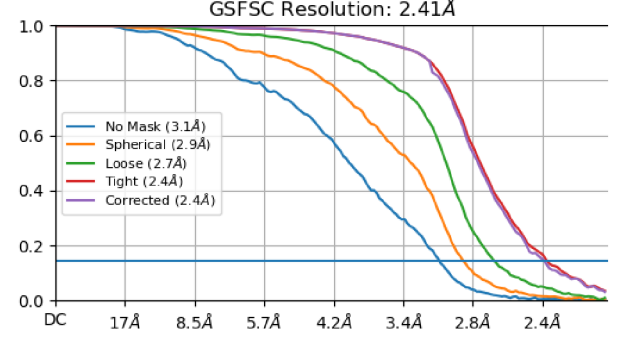

Fig. S3. FSC curves for $70 S$ ribosomes under various conditions 

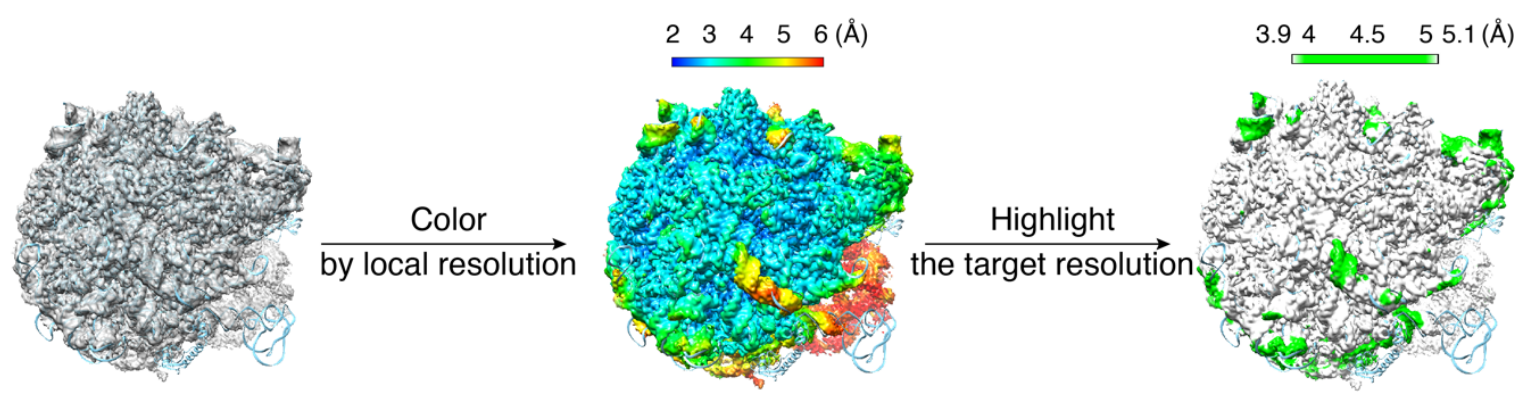

Zone target regions
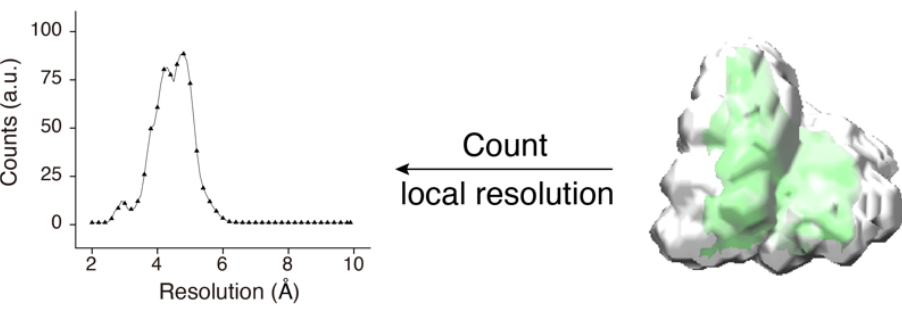

Make mask for similar volume in different structures

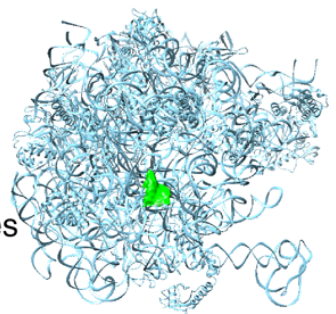

\section{Fig. S4. Flow chart for local resolution calculation on selected regions}

272 To compare local resolutions from different structures, the volumes of the target regions in different EM maps were adjusted to the same level via changing thresholds. 

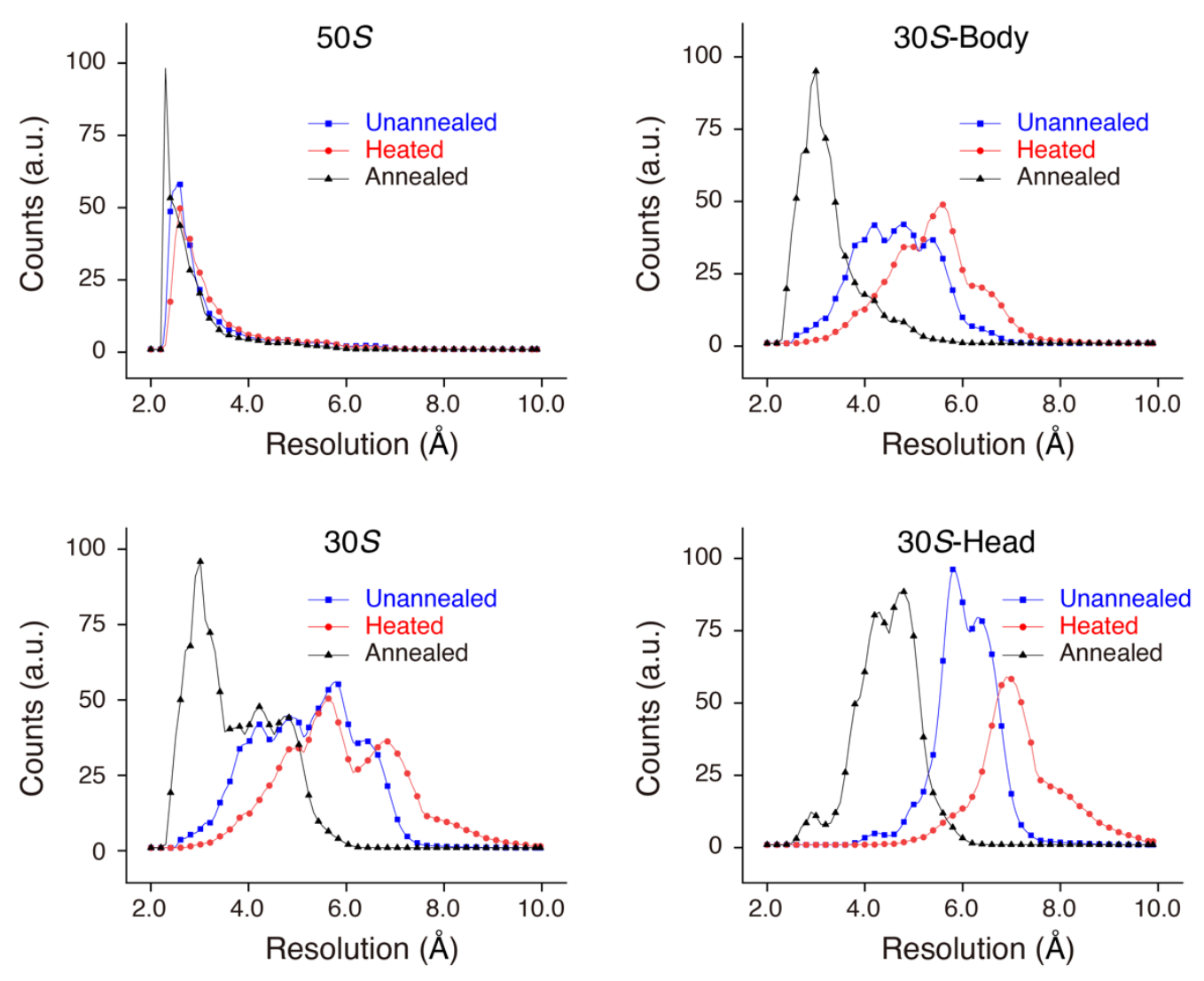

276 Fig. S5. Local resolution histograms of different subdomains from the unannealed, heated, 277 and annealed $70 S$ ribosomes

278 The mean and standard deviation from these histograms were calculated (Fig. 1B). "a.u." means 279 arbitrary units. 

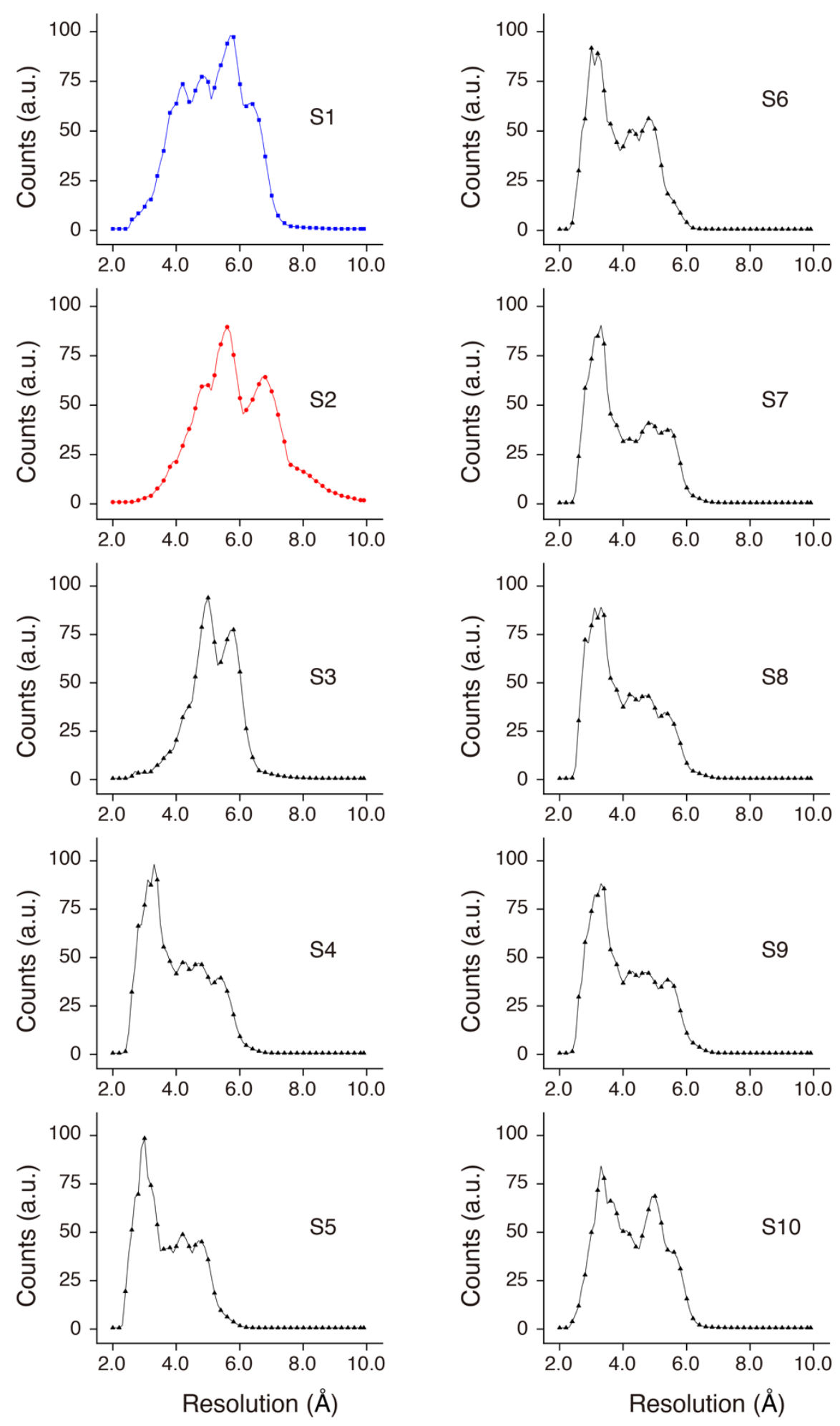

Fig. S6. Local resolution histograms of $30 S$ subunits under various conditions The mean and standard deviation from these histograms were calculated (Fig. 2C). 
A

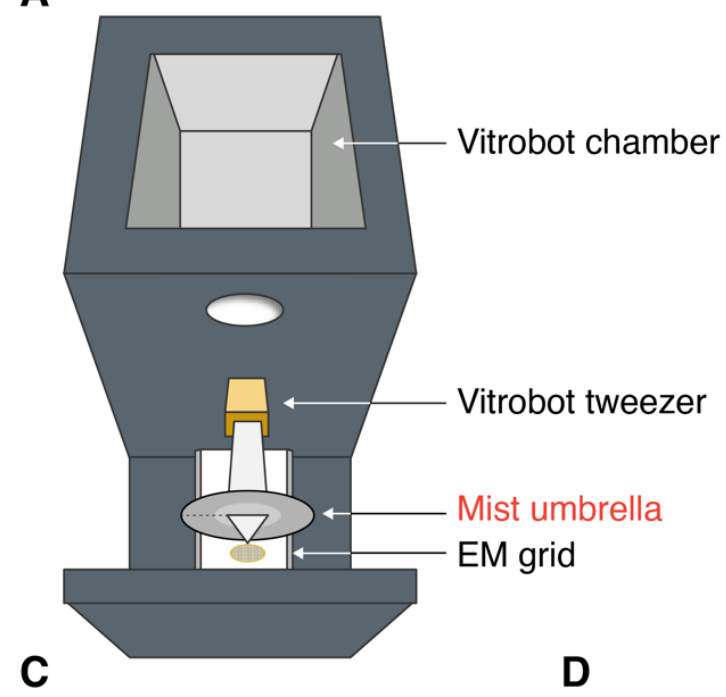

B

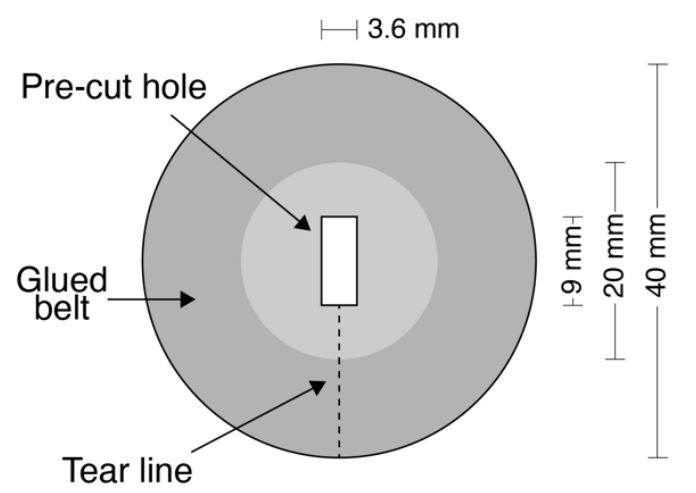

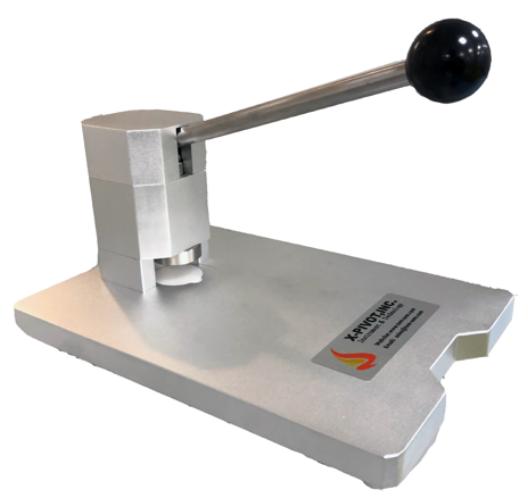
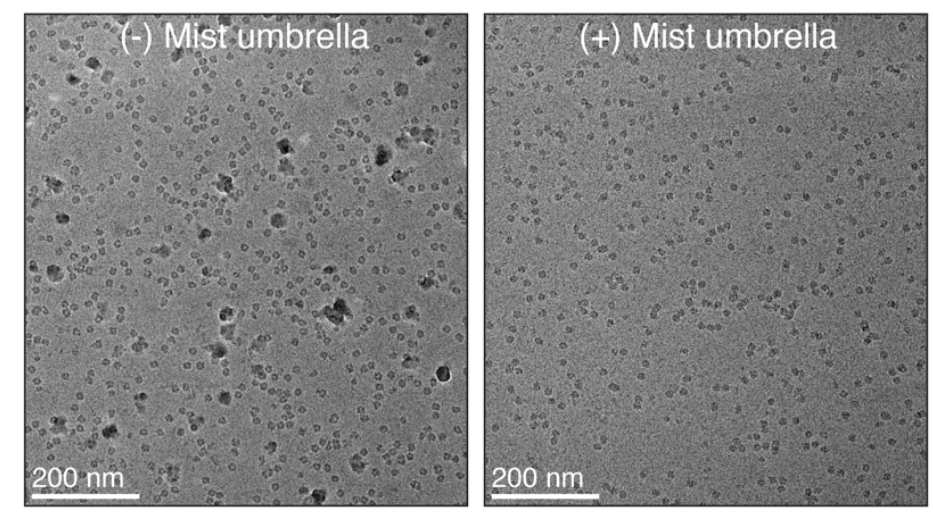

E
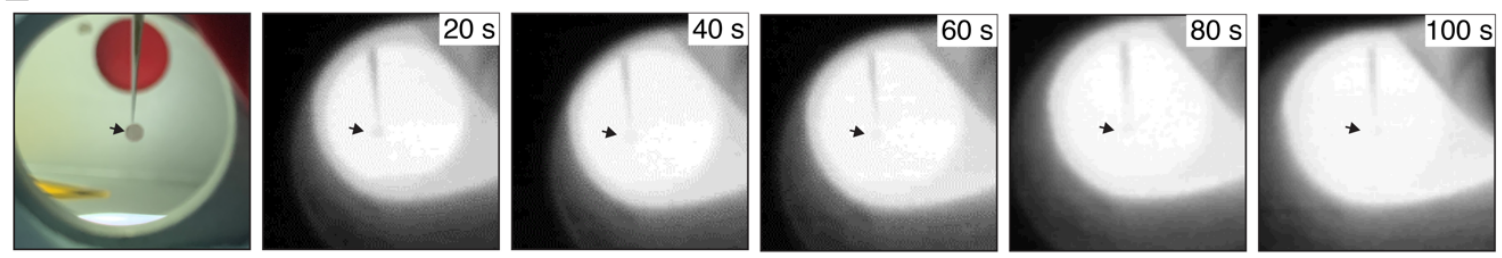

Fig. S7. Optimization for temperature-dependent cryo-EM

(A) Diagram for the Vitrobot device with a mist umbrella. (B) Detailed parameters for the mist umbrella. Briefly, there is a pre-cut hole on round filter paper. (C) Home-made device to fabricate mist umbrellas. (D) Cryo-EM micrographs with or without a mist umbrella. (E) Heat exchange between grids and the Vitrobot chamber reached a balance in $\sim 100 \mathrm{~s}$. Black arrow points to the position of the cryo-EM grid. 


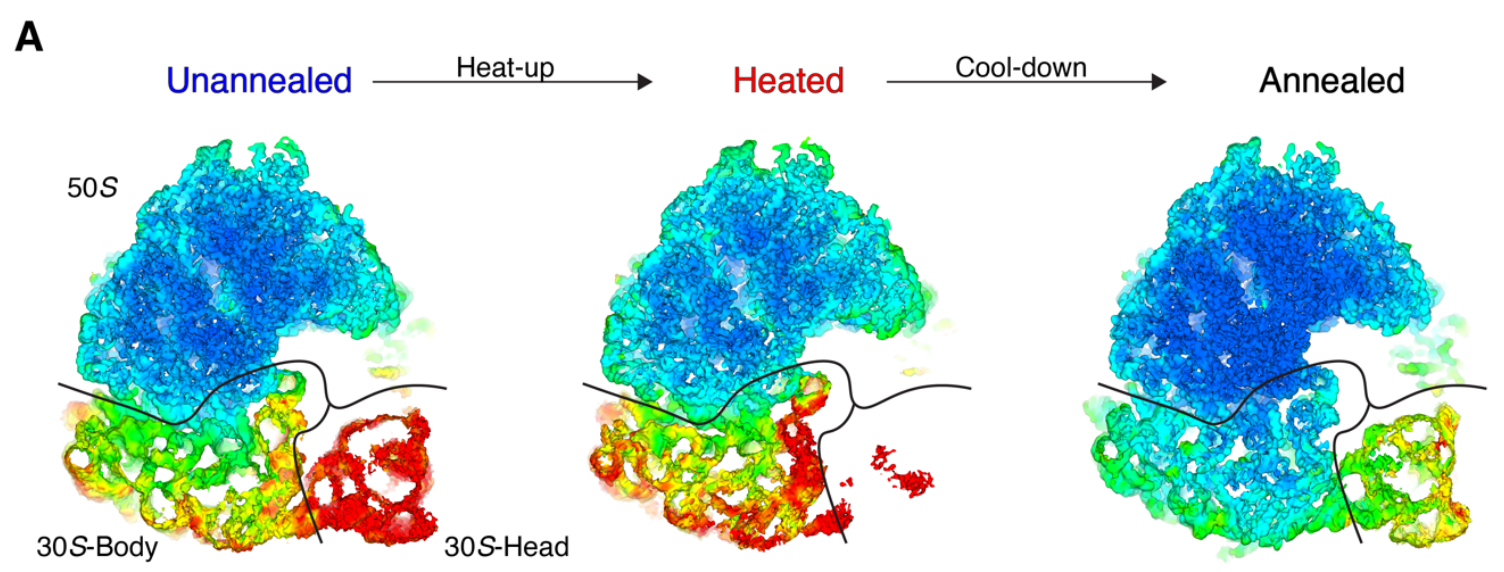

B Unannealed

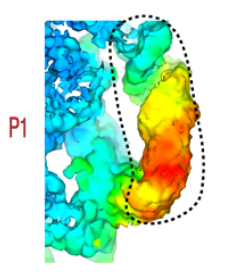

C
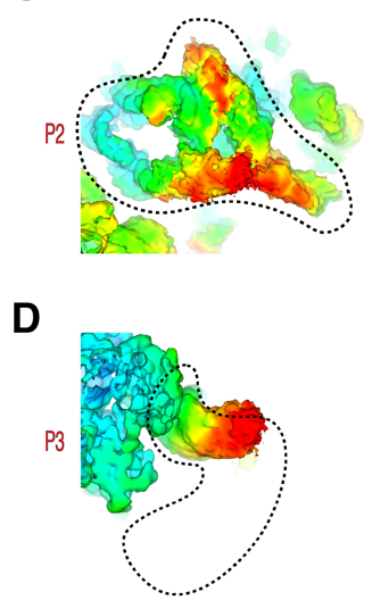
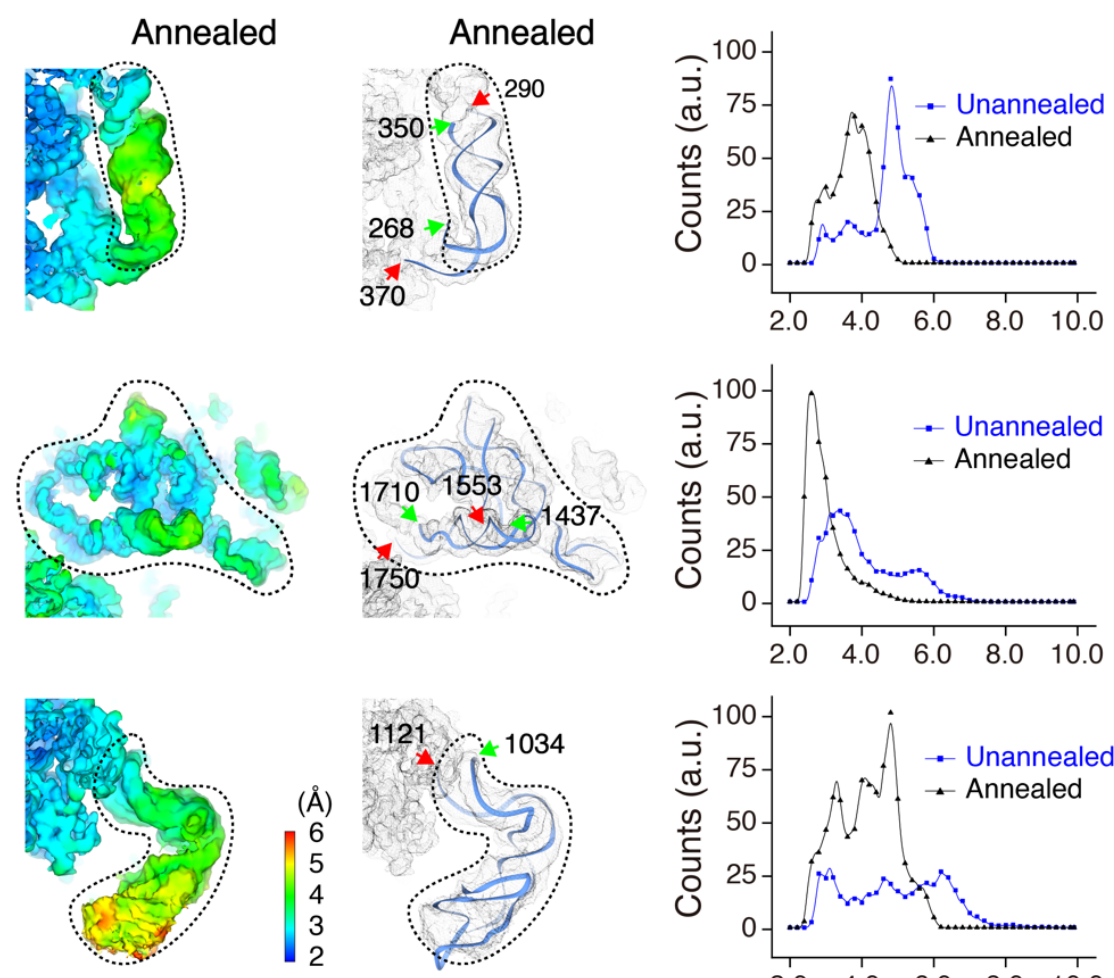

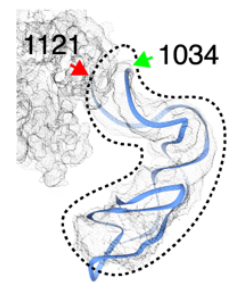

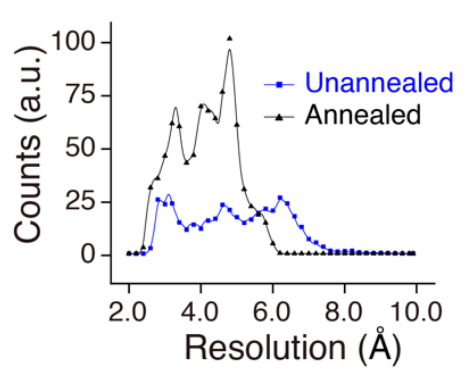

Fig. S8. Annealing stabilizes flexible regions on the periphery of the $50 S$ subunit

(A) Central slice of local resolution maps of the unannealed, heated, and annealed $70 S$ ribosomes. (B-D) Annealing stabilizes three typical flexible regions on the periphery of the $50 \mathrm{~S}$ subunit. The positions of $\mathrm{P} 1, \mathrm{P} 2$, and $\mathrm{P} 3$ in $70 S$ ribosomes are shown in Fig. 1A. Local resolution maps and histograms in the unannealed and annealed conditions are shown. Models were based on the annealed structures. Green and red arrows indicate the start and end, respectively, of selected strands. 


\section{A}
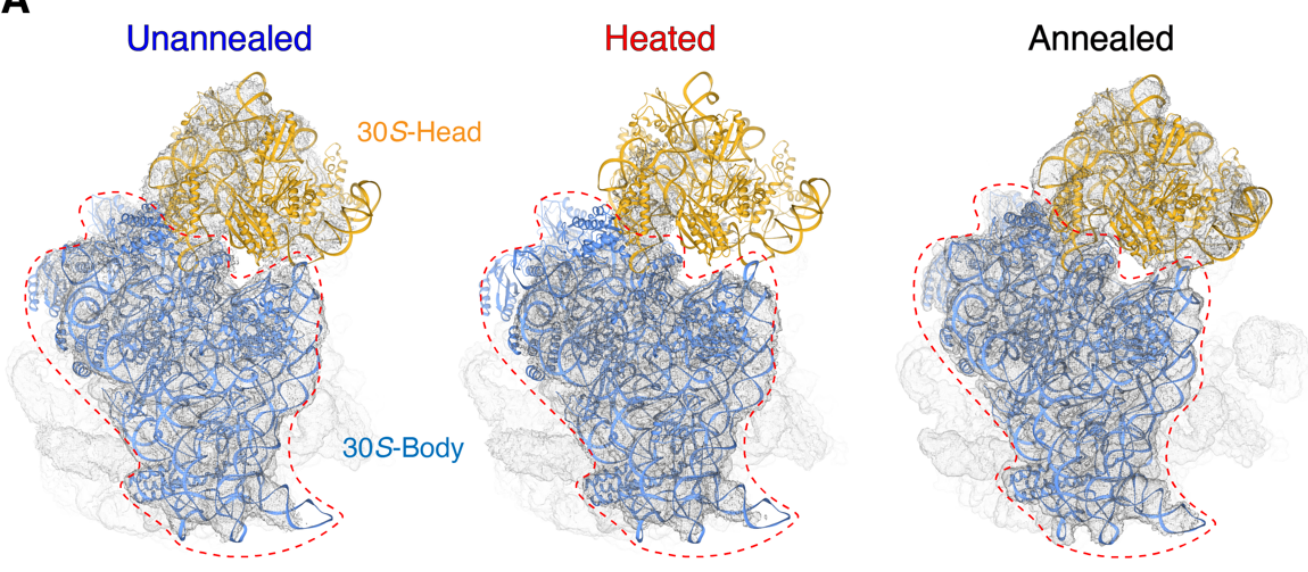

B

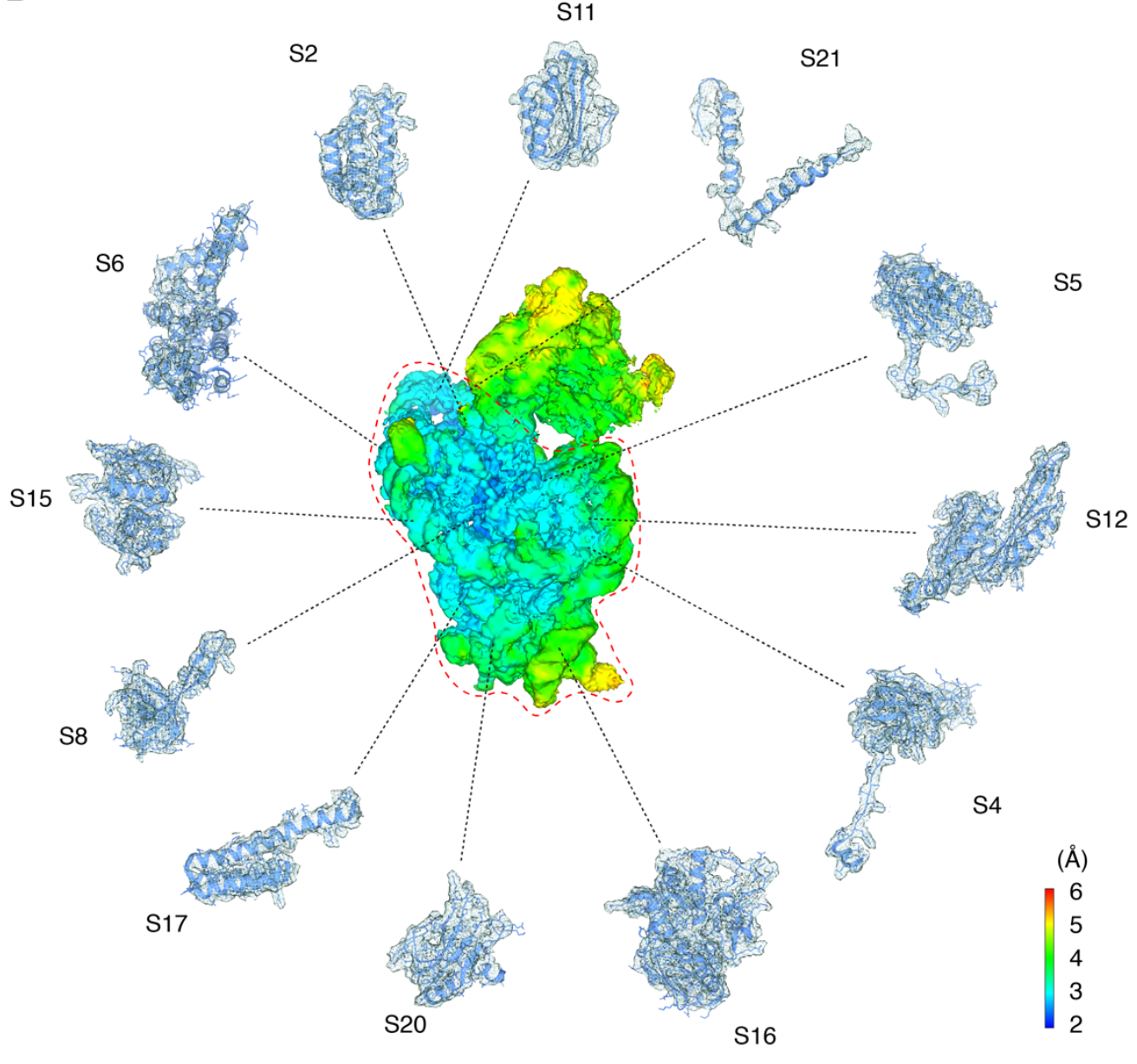

Fig. S9. Modeling of $30 S$ subunits from the unannealed, heated, and annealed ribosomes

(A) Docking of the atomic model of the $30 S$ subunit into the unannealed, heated, and annealed ribosomes. The body domains of the $30 S$ subunit were marked for high docking accuracy in all ribosomes. (B) Expanded view of peripheral proteins fitting into the body domain of the annealed $30 S$ subunit. 
A
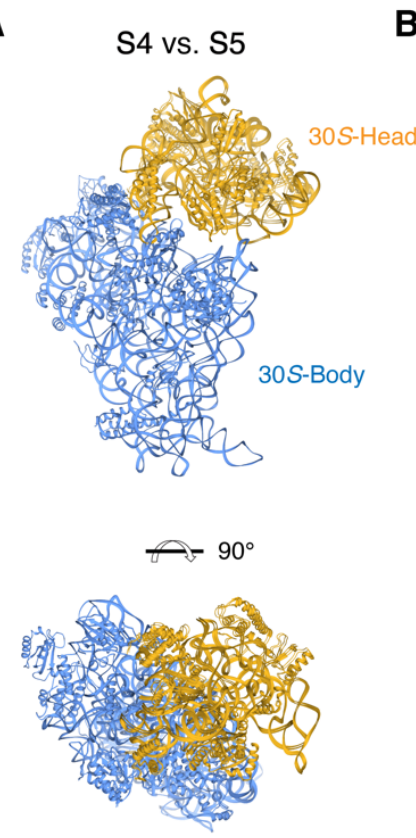

E
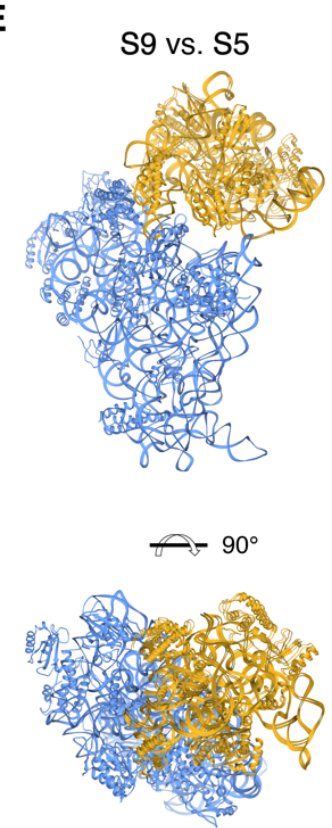

B
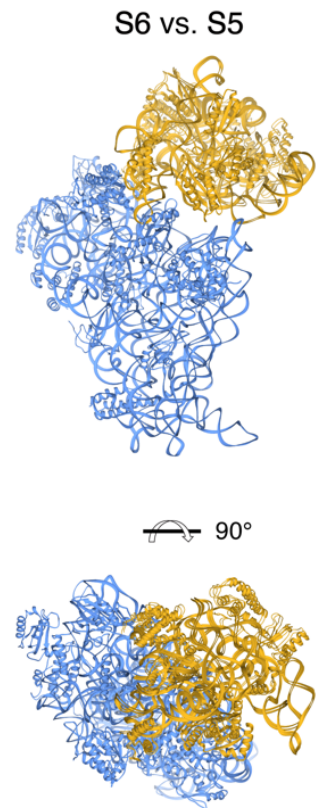

$\mathbf{F}$
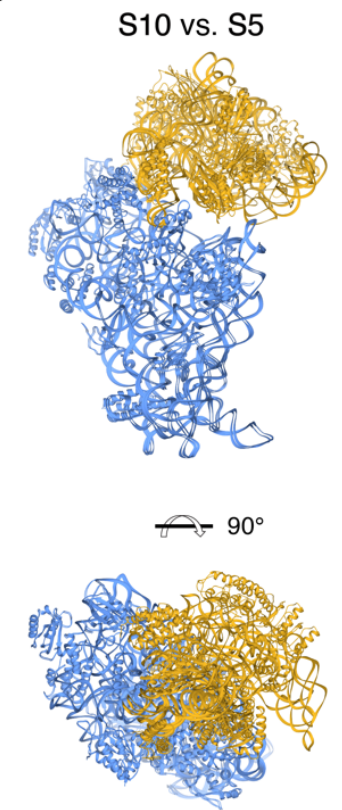

C
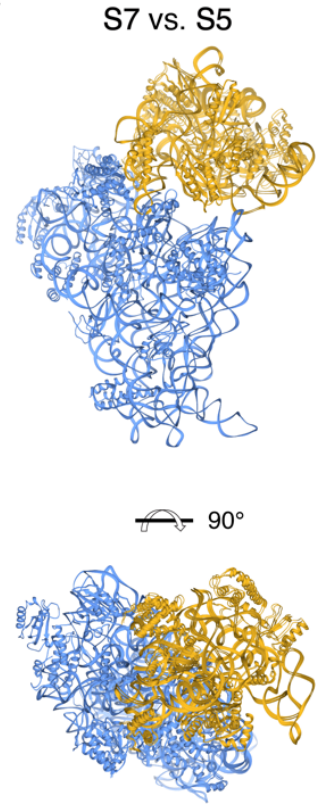

G

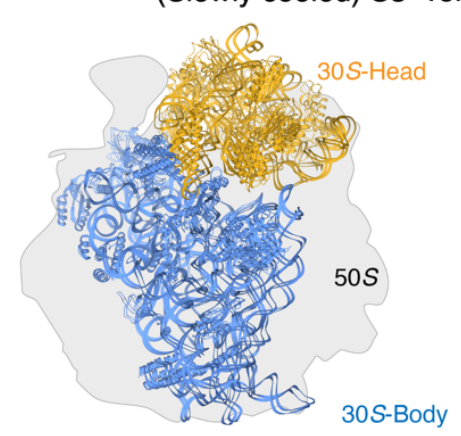

(Slowly cooled) S3 vs. S1 (Unannealed)
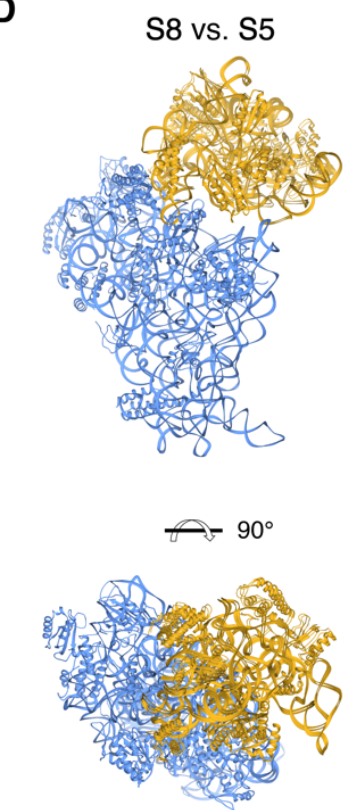

Fig. S10. Rotational comparison of $30 S$ subunits under various annealing conditions

(A-F) $50 S$ subunits under various annealing conditions were aligned as the reference, and atomic models for the $30 S$ subunits are shown. S1, S3, and S4-S10 correspond to annealing conditions as listed in Fig. 2B. (G) Rotational comparison of $30 S$ subunits between slowly cooled and unannealed ribosomes. Left: Atomic models for the $30 S$ subunits. Right: Difference vectors 314 between phosphorous and $\mathrm{C} \alpha$ atoms in the $30 S$ subunits. 


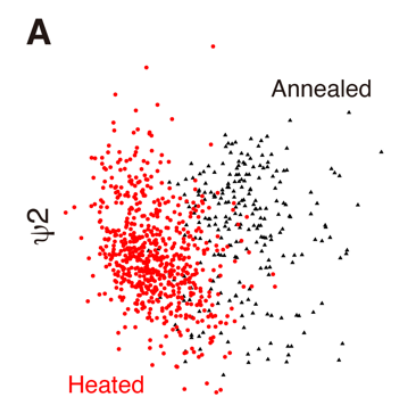

$\psi 1$

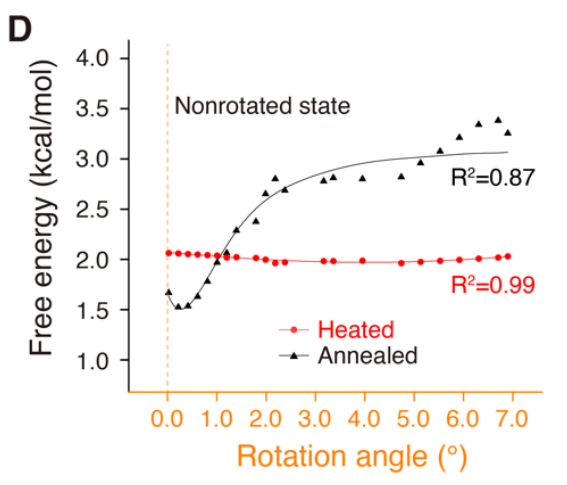

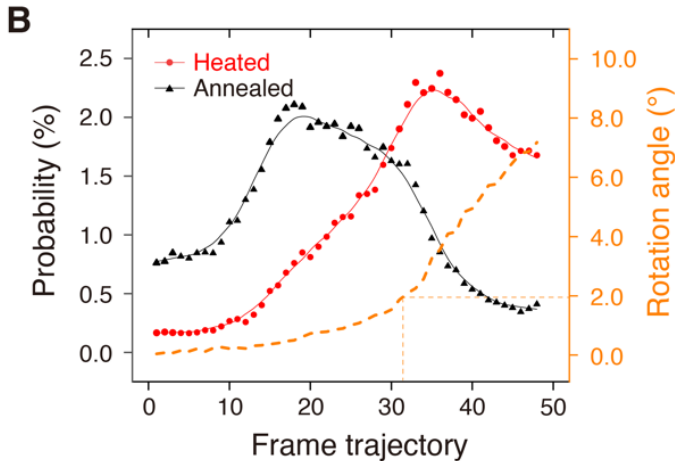

E

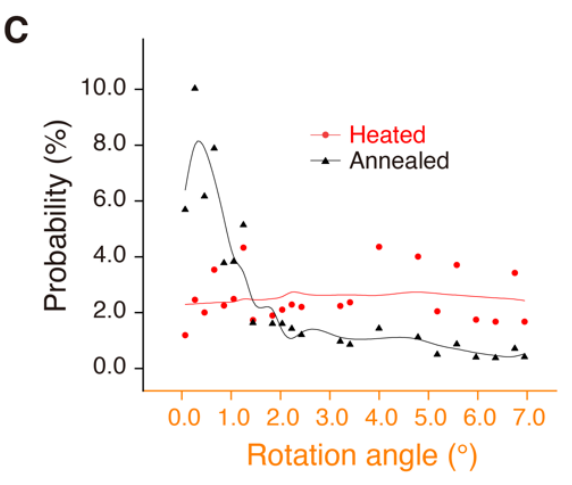

Fig. S11. Free-energy minimization occurs during cooling from the heated ribosome to the annealed ribosome

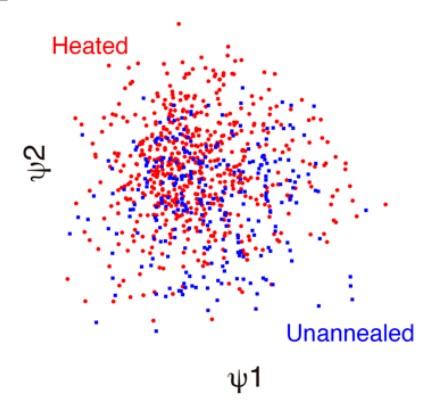

(A) Initial manifold snapshots of the $70 S$ ribosome in one projection direction. The points are colored in accordance with the heated and annealed subsets. The projection direction is approximately orthogonal to the interface between the $50 S$ and $30 S$ subunits. (B) Particle distribution of the heated and annealed ribosomes along the frame trajectory. The 3D structure at each frame was reconstructed, and the rotation angle of $30 S$ subunit with respect to the nonrotated state was calculated. (C) Particle distribution of the heated and annealed ribosomes along the rotation angle. Particle distribution was recalculated with the rotation angle at an interval of $0.2^{\circ}$, and a moving average was used to smooth the data variation. (D) Free-energy distribution of the heated and annealed ribosomes along the rotation angle. The free energy was calculated from the fitted curve in $(\mathbf{C})$. (E) Initial manifold snapshots of the $70 S$ ribosome in one projection direction. The points are colored in accordance with the unannealed and heated subsets. It is challenging to efficiently separate two states. 

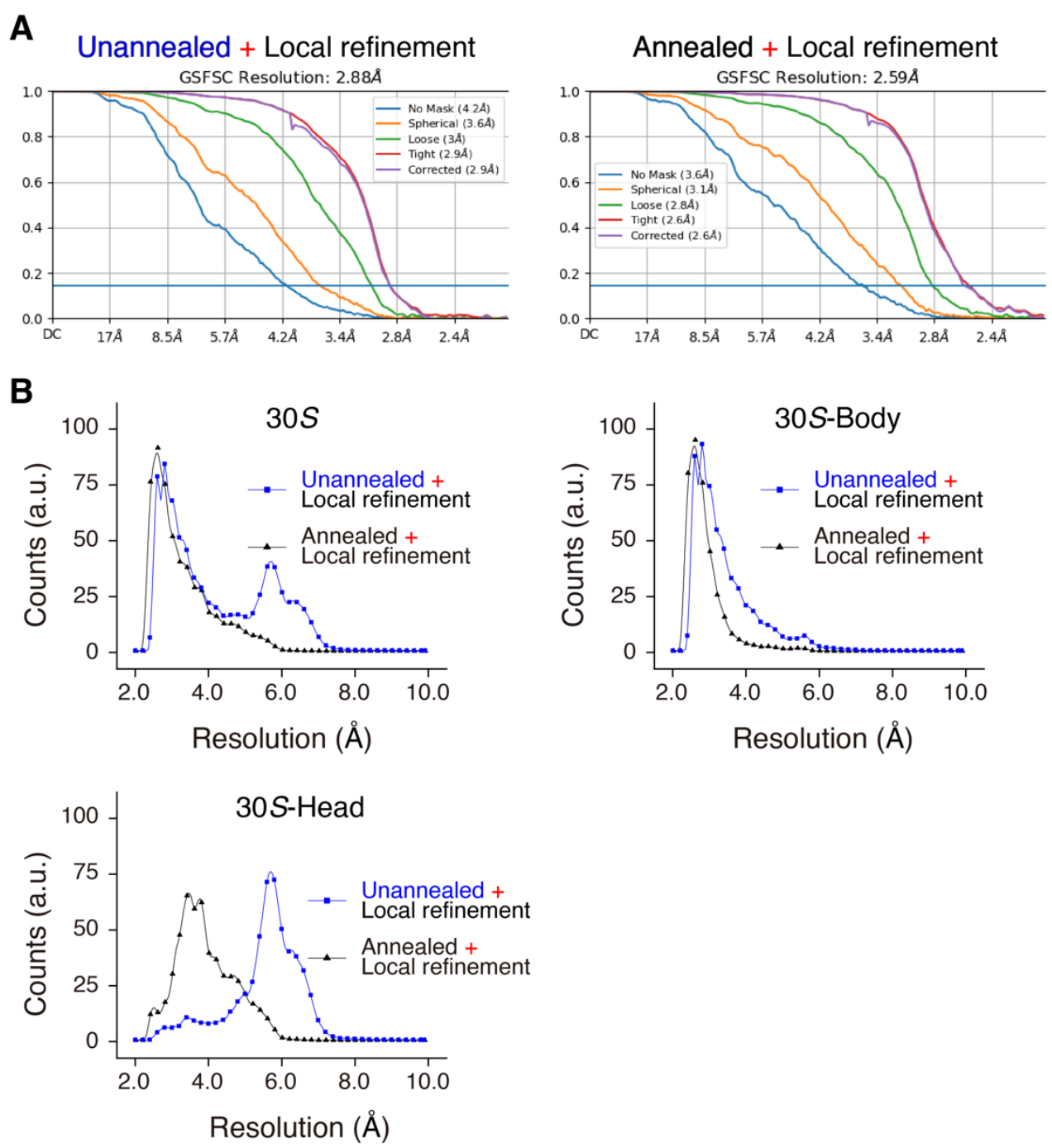

\section{Fig. S12. Resolution estimation of $30 S$ subunits after local refinements}

333 (A) FSC curves for $30 S$ subunits after local refinements on unannealed and annealed ribosomes. (B) Local resolution histograms of the $30 S$ subunit and subdomains after local refinements on unannealed and annealed ribosomes. 
A $\begin{array}{lll}\text { Unannealed } & \text { Unannealed }+ \\ \text { Local refinement } & \text { Annealed } & \begin{array}{c}\text { Annealed }+ \\ \text { Local refinement }\end{array}\end{array}$
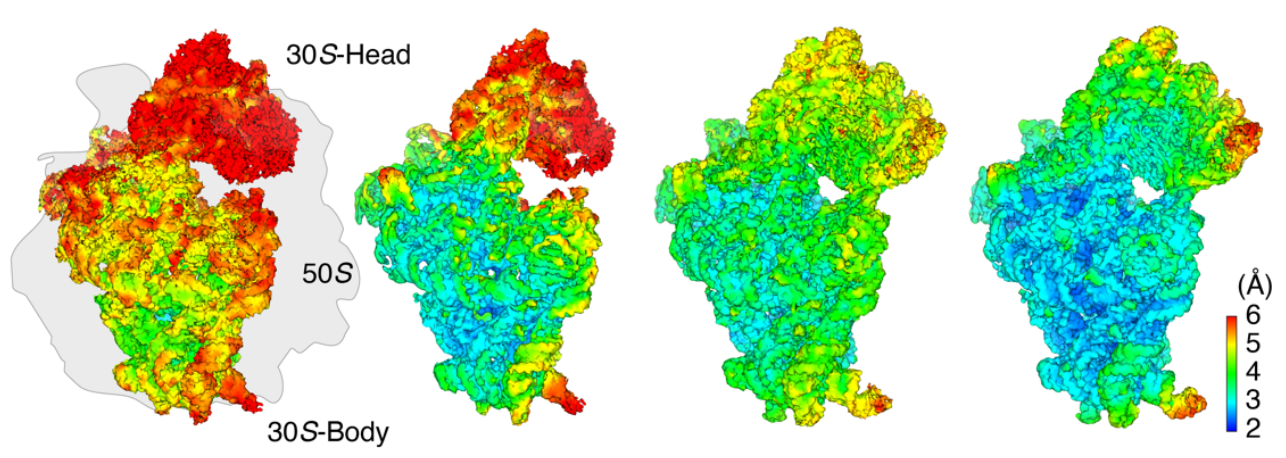

B

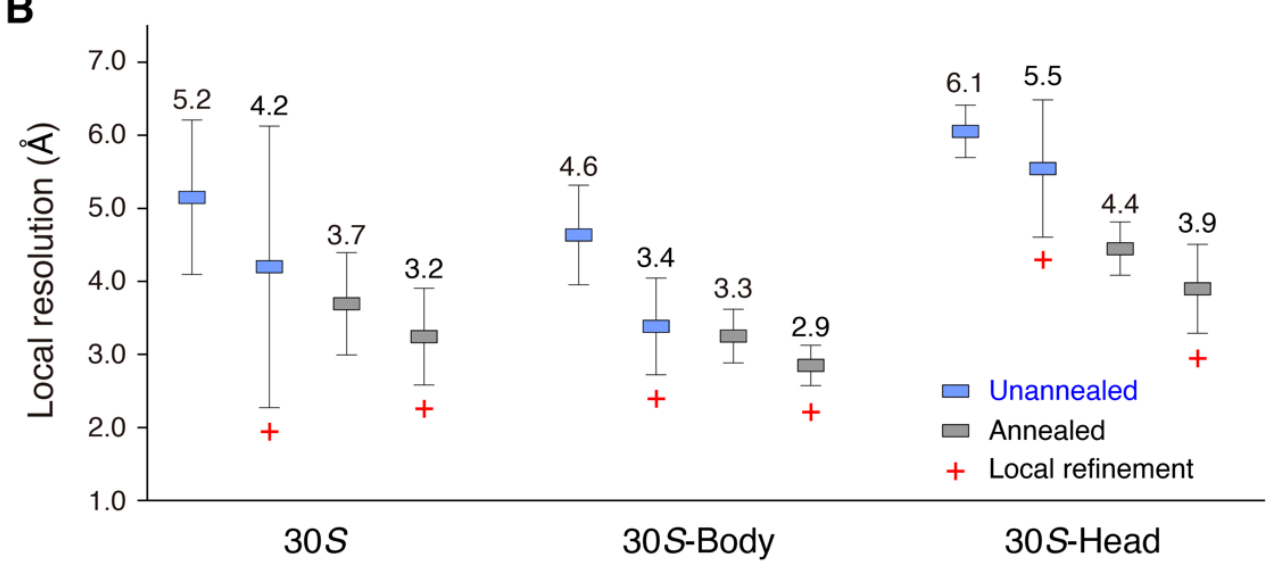

338 Fig. S13. Structural comparison of $\mathbf{3 0 S}$ subunits before/after local refinements

339 (A) Local resolution maps of $30 S$ subunits under various conditions. (B) Local resolution 340 comparison of unannealed and annealed $30 S$ subunits before/after local refinements. 
Table S1. Cryo-EM data collection and data processing statistics

\begin{tabular}{|c|c|c|}
\hline & $\begin{array}{l}\text { Heated ribosome } \\
\text { (S2) }\end{array}$ & $\begin{array}{l}\text { Unannealed (S1) } \\
\text { and annealed (S3- } \\
\text { S10) ribosomes }\end{array}$ \\
\hline \multicolumn{3}{|l|}{ Sample preparation } \\
\hline Concentration (nM) & 700 & 700 \\
\hline Loading volume $(\mu \mathrm{L})$ & 4.5 & 4 \\
\hline Chamber temperature $\left({ }^{\circ} \mathrm{C}\right)$ & 37 & 4 \\
\hline Chamber humidity (\%) & 100 & 100 \\
\hline Blot time (s) & 0.5 & 0.5 \\
\hline Blot force & -5 & -2 \\
\hline \multicolumn{3}{|l|}{ Data collection and processing } \\
\hline Microscope & \multicolumn{2}{|c|}{ Titan Krios $\mathrm{G}^{3 \mathrm{i}}$} \\
\hline Voltage $(\mathrm{kV})$ & \multicolumn{2}{|c|}{300} \\
\hline Camera & \multicolumn{2}{|c|}{ Gatan K3 BioQuantum } \\
\hline Magnification & \multicolumn{2}{|c|}{81,000} \\
\hline Electron exposure $\left(\mathrm{e}^{-} / \AA^{2}\right)$ & \multicolumn{2}{|c|}{60} \\
\hline Defocus range $(\mu \mathrm{m})$ & \multicolumn{2}{|c|}{$1-1.5$} \\
\hline Pixel size $(\AA)$ & \multicolumn{2}{|c|}{0.53} \\
\hline Symmetry imposed & \multicolumn{2}{|c|}{ C1 } \\
\hline Initial particle images (no.) & \multicolumn{2}{|c|}{ Fig. S2b } \\
\hline Final particle images (no.) & \multicolumn{2}{|c|}{ Fig. S2b } \\
\hline Map resolution $(\AA)$ & \multicolumn{2}{|c|}{ Fig. S3 } \\
\hline FSC threshold & \multicolumn{2}{|c|}{0.143} \\
\hline Map resolution range $(\AA)$ & \multicolumn{2}{|c|}{ Fig. S6 } \\
\hline
\end{tabular}


Movie S1. Cartoon that illustrates how to use the mist umbrella ribosomes

\section{References}

1. J. Lin, M. G. Gagnon, D. Bulkley, T. A. Steitz, Conformational changes of elongation factor $\mathrm{G}$ on the ribosome during tRNA translocation. Cell 160, 219-227 (2015).

2. G. Blaha et al., Preparation of functional ribosomal complexes and effect of buffer conditions on tRNA positions observed by cryoelectron microscopy. Methods Enzymol 317, 292-309 (2000).

3. R. F. Thompson, M. G. Iadanza, E. L. Hesketh, S. Rawson, N. A. Ranson, Collection, pre-processing and on-the-fly analysis of data for high-resolution, single-particle cryoelectron microscopy. Nat Protoc 14, 100-118 (2019).

4. A. Punjani, J. L. Rubinstein, D. J. Fleet, M. A. Brubaker, cryoSPARC: algorithms for rapid unsupervised cryo-EM structure determination. Nature methods 14, 290-296 (2017).

5. S. Q. Zheng et al., MotionCor2: anisotropic correction of beam-induced motion for improved cryo-electron microscopy. Nature methods 14, 331-332 (2017).

6. A. Rohou, N. Grigorieff, CTFFIND4: Fast and accurate defocus estimation from electron micrographs. Journal of structural biology 192, 216-221 (2015).

7. N. R. James, A. Brown, Y. Gordiyenko, V. Ramakrishnan, Translational termination without a stop codon. Science 354, 1437-1440 (2016).

8. S. Lindert, J. Meiler, J. A. McCammon, Iterative Molecular Dynamics-Rosetta Protein Structure Refinement Protocol to Improve Model Quality. Journal of chemical theory and computation 9, 3843-3847 (2013).

9. P. Emsley, B. Lohkamp, W. G. Scott, K. Cowtan, Features and development of Coot. Acta crystallographica. Section D, Biological crystallography 66, 486-501 (2010).

10. P. D. Adams et al., PHENIX: a comprehensive Python-based system for macromolecular structure solution. Acta crystallographica. Section D, Biological crystallography 66, 213221 (2010).

11. J. A. Dunkle et al., Structures of the bacterial ribosome in classical and hybrid states of tRNA binding. Science 332, 981-984 (2011).

12. W. Zhang, J. A. Dunkle, J. H. Cate, Structures of the ribosome in intermediate states of ratcheting. Science 325, 1014-1017 (2009).

13. E. F. Pettersen et al., UCSF Chimera--a visualization system for exploratory research and analysis. Journal of computational chemistry 25, 1605-1612 (2004).

14. A. Kucukelbir, F. J. Sigworth, H. D. Tagare, Quantifying the local resolution of cryo-EM density maps. Nat Methods 11, 63-65 (2014).

15. N. Fischer, A. L. Konevega, W. Wintermeyer, M. V. Rodnina, H. Stark, Ribosome dynamics and tRNA movement by time-resolved electron cryomicroscopy. Nature 466, 329-333 (2010). 
389 16. A. Dashti et al., Trajectories of the ribosome as a Brownian nanomachine. Proc. Natl. Acad. Sci. U. S. A. 111, 17492-17497 (2014). 